\title{
CRITICALITY OF THE EXPONENTIAL RATE OF DECAY FOR THE LARGEST NEAREST-NEIGHBOR LINK IN RANDOM GEOMETRIC GRAPHS
}

\author{
BHUPENDER GUPTA, * Indian Institute of Information Technology \\ SRIKANTH K. IYER, ${ }^{* *}$ Indian Institute of Science
}

\begin{abstract}
Let $n$ points be placed independently in $d$-dimensional space according to the density $f(x)=A_{d} \mathrm{e}^{-\lambda\|x\|^{\alpha}}, \lambda, \alpha>0, x \in \mathbb{R}^{d}, d \geq 2$. Let $d_{n}$ be the longest edge length of the nearest-neighbor graph on these points. We show that $\left(\lambda^{-1} \log n\right)^{1-1 / \alpha} \mathrm{d}_{n}-$ $b_{n}$ converges weakly to the Gumbel distribution, where $b_{n} \sim((d-1) / \lambda \alpha) \log \log n$. We also prove the following strong law for the normalized nearest-neighbor distance $\tilde{d}_{n}=\left(\lambda^{-1} \log n\right)^{1-1 / \alpha} \mathrm{d}_{n} / \log \log n:(d-1) / \alpha \lambda \leq \liminf _{n \rightarrow \infty} \tilde{d}_{n} \leq \lim _{\sup _{n \rightarrow \infty}} \tilde{d}_{n} \leq$ $d / \alpha \lambda$ almost surely. Thus, the exponential rate of decay $\alpha=1$ is critical, in the sense that, for $\alpha>1, d_{n} \rightarrow 0$, whereas, for $\alpha \leq 1, d_{n} \rightarrow \infty$ almost surely as $n \rightarrow \infty$.
\end{abstract}

Keywords: Random geometric graph; nearest-neighbor graph; Poisson point process; largest nearest-neighbor link; vertex degree

2010 Mathematics Subject Classification: Primary 60D05; 60G70

Secondary $05 \mathrm{C} 05 ; 90 \mathrm{C} 27$

\section{Introduction and main results}

In this paper we prove weak and strong laws for the largest nearest-neighbor distance of points distributed according to the probability density function

$$
f(x)=A_{d} \mathrm{e}^{-\lambda\|x\|^{\alpha}}, \quad \lambda>0, \alpha>0, x \in \mathbb{R}^{d}, d \geq 2,
$$

where $\|\cdot\|$ is the Euclidean $\left(\ell_{2}\right)$ norm on $\mathbb{R}^{d}$ and

$$
A_{d}=\frac{\alpha \lambda^{d / \alpha} \Gamma(d / 2+1)}{d \pi^{d / 2} \Gamma(d / \alpha)} .
$$

If $X$ has density given by (1.1) then $R=\|X\|$ has density,

$$
f_{R}(r)=A_{d}^{\prime} r^{d-1} \mathrm{e}^{-\lambda r^{\alpha}}, \quad 0<r<\infty, d \geq 2,
$$

where $A_{d}^{\prime}=\alpha \lambda^{d / \alpha} / \Gamma(d / \alpha)$. The basic object of study will be the graphs $G_{n}$ with vertex set $\mathcal{X}_{n}=\left\{X_{1}, X_{2}, \ldots, X_{n}\right\}, n=1,2, \ldots$, where the vertices are independently distributed according to $f$. Edges of $G_{n}$ are formed by connecting each of the vertices in $\mathcal{X}_{n}$ to its nearest

Received 17 June 2006; revision received 18 March 2010.

* Postal address: Department of Computer Science and Engineering, Indian Institute of Information Technology, Jabalpur 482011, India.

** Postal address: Department of Mathematics, Indian Institute of Science, Bangalore 560012, India.

Email address: skiyer@math.iisc.ernet.in

Research supported in part by UGC SAP IV and a grant from the DRDO-IISc program on Mathematical Engineering. 
neighbor. The longest edge of the graph $G_{n}$ is denoted by $d_{n}$. We will refer to $G_{n}$ as the nearestneighbor graph (NNG) on $\mathcal{X}_{n}$ and to $d_{n}$ as the largest nearest-neighbor distance (LNND). For any finite subset $\mathcal{X} \subset \mathbb{R}^{d}$, let $G(\mathcal{X}, r)$ denote the graph with vertex set $\mathcal{X}$ and edges between all pairs of vertices that are at distances less than $r$. Thus, $d_{n}$ is the minimum $r_{n}$ required so that the graph $G\left(\mathcal{X}_{n}, r_{n}\right)$ has no isolated nodes.

The largest nearest-neighbor link has been studied in the context of computational geometry (see Dette and Henze (1989) and Steele and Tierney (1986)) and has applications in statistics, computer science, biology, and the physical sciences. For a detailed description of random geometric graphs, their properties, and applications, we refer the reader to Penrose (2003) and the references therein.

The asymptotic distribution of $d_{n}$ was derived in Penrose (1997), assuming that $f$ is uniform on the unit cube. It is shown that if the metric is assumed to be the toroidal, and if $\theta$ is the volume of the unit ball, then $n \theta d_{n}^{d}-b_{n}$ converges weakly to the Gumbel distribution, where $b_{n} \sim$ $\log n$. Penrose (1998) showed that, for normally distributed points $(\alpha=2), \sqrt{(2 \log n)} \mathrm{d}_{n}-b_{n}$ converges weakly to the Gumbel distribution, where $b_{n} \sim(d-1) \log \log n$. The above result is also shown to be true for the longest edge of the minimal spanning tree. The notation $a_{n} \sim b_{n}$ implies that $a_{n} / b_{n}$ converges to 1 as $n \rightarrow \infty$. Hsing and Rootzén (2005) derived the asymptotic distribution for $d_{n}$ in the case $d=2$, for a large class of densities, including elliptically contoured distributions, distributions with independent Weibull-like marginals, and distributions with parallel level curves (which includes the densities defined by (1.1)). Appel and Russo (1997) proved strong laws for $d_{n}$ for graphs on uniform points in the $d$-dimensional unit cube. Penrose (1999) extended this to general densities having compact support $\Omega$ for which $\min _{x \in \Omega} f(x)>0$.

Our aim in this paper is to show that when the tail of the density decays like an exponential or slower $(\alpha \leq 1), d_{n}$ diverges, whereas, for superexponential decay of the tail, $d_{n} \rightarrow 0$, almost surely (a.s.) as $n \rightarrow \infty$. Properties of one-dimensional exponential random geometric graphs have been studied in Gupta et al. (2005). In this case, spacings between the ordered nodes are independent and exponentially distributed. This allows for explicit computations of many characteristics for the graph and both strong and weak laws can be established.

It is often easier to study the graph $G_{n}$ via the NNG $P_{n}$ on the set $\mathcal{P}_{n}=\left\{X_{1}, X_{2}, \ldots, X_{N_{n}}\right\}$, $n \geq 1$, where $\left\{N_{n}\right\}_{n \geq 1}$ is a sequence of Poisson random variables that are independent of the sequence $\left\{X_{n}\right\}_{n \geq 1}$ with $\mathrm{E}\left[N_{n}\right]=n$. Here $\mathcal{P}_{n}$ is an inhomogeneous Poisson point process with intensity function $n f(\cdot)$ (see Penrose (2003, Proposition 1.5)). Note that the graphs $G_{n}$ and $P_{n}$ are coupled, since the first $\min \left(n, N_{n}\right)$ vertices of the two graphs are identical. We also assume that the random variables $N_{n}$ are nondecreasing, so that $\mathcal{P}_{1} \subset \mathcal{P}_{2} \subset \mathcal{P}_{3} \subset \cdots$.

Let $W_{n}\left(r_{n}\right)$ and $W_{n}^{\prime}\left(r_{n}\right)$ be the numbers of vertices of degree 0 (isolated nodes) in $G\left(\mathcal{X}_{n}, r_{n}\right)$ and $G\left(\mathcal{P}_{n}, r_{n}\right)$, respectively. Let $\theta_{d}$ denote the volume of the $d$-dimensional unit ball in $\mathbb{R}^{d}$, and let $\operatorname{Po}(\lambda)$ denote a Poisson distribution with mean $\lambda>0$. In what follows we will write $\log _{2} n$ for $\log \log n$ and $\log _{3} n$ for $\log \log \log n$, etc.

For any $\beta \in \mathbb{R}$, let $\left\{r_{n}\right\}_{n \geq 1}$ be a sequence of edge distances that satisfies

$$
r_{n}\left(\lambda^{-1} \log n\right)^{1-1 / \alpha}-\frac{d-1}{\lambda \alpha} \log _{2} n+\frac{d-1}{2 \lambda \alpha} \log _{3} n \rightarrow \frac{\beta}{\lambda \alpha}
$$

as $n \rightarrow \infty$. We now state our main results.

Theorem 1.1. Let $\left\{r_{n}\right\}_{n \geq 1}$ satisfy (1.4) as $n \rightarrow \infty$. Then

$$
W_{n}\left(r_{n}\right) \rightarrow \operatorname{Po}\left(\frac{\mathrm{e}^{-\beta}}{C_{d}}\right)
$$


in distribution, where

$$
C_{d}=\frac{\alpha^{1-d} \theta_{d-1}(d-1) !}{2}\left(\frac{d-1}{2 \pi}\right)^{(d-1) / 2} .
$$

An easy consequence of the above result is the following limiting distribution for $d_{n}$.

Theorem 1.2. Let $f(\cdot)$ be the d-dimensional density defined in (1.1). Let $d_{n}$ be the largest nearest-neighbor link of the graph $G_{n}$ of $n$ independent and identically distributed points $X_{n}=\left\{X_{1}, X_{2}, \ldots, X_{n}\right\}$ distributed according to $f$. Then, for any $\gamma \in \mathbb{R}$,

$\lim _{n \rightarrow \infty} \mathrm{P}\left[\lambda \alpha\left(\lambda^{-1} \log n\right)^{1-1 / \alpha} \mathrm{d}_{n}-(d-1) \log _{2} n+\frac{d-1}{2} \log _{3} n \leq \gamma-\log \left(C_{d}\right)\right] \rightarrow \exp \left(-\mathrm{e}^{-\gamma}\right)$.

The above result for the case $\alpha=2$ was derived in Penrose (1998). In dimension $d=2$, Theorem 1.2 follows from Theorem 7 of Hsing and Rootzén (2005) (see also Example 3 therein). Their method is based on spatial blocking and uses a locally orthogonal coordinate system with respect to the level curves. We follow the approach in Penrose (1998) and use the Chen-Stein method.

Strong laws exist in the literature only for densities that do not vanish and whose support is bounded. Suppose that $d \geq 2$, the density $f$ is continuous, has support $\Omega$, and that the boundary $\partial \Omega$ is a compact $(d-1)$-dimensional $C^{2}$ submanifold of $\mathbb{R}^{d}$. Let $f_{0}>0$ be the essential infimum of $f$ restricted to $\Omega$, and let $f_{1}=\inf _{\partial \Omega} f$. Then (see Theorem 7.2 of Penrose (2003)), $\lim _{n \rightarrow \infty} n d_{n}^{d} / \log n=\max \left\{c_{0} f_{0}^{-1}, c_{1} f_{1}^{-1}\right\}$ a.s. Thus, the asymptotic behavior of the LNND depends on the (reciprocal of the) infimum of the density, since it is in the vicinity of this infimum that points will be sparse and, hence, be farthest from each other. If $f_{0}$ or $f_{1}$ is 0 , then the right-hand side is infinite, implying that the scaling on the left is not the appropriate one. We now state a strong law for the LNND in our case.

Theorem 1.3. Let $d_{n}$ be the $L N N D$ of the $N N G G_{n}$ defined on the collection $\mathcal{X}_{n}$ of $n$ points distributed independently and identically according to the density $f(\cdot)$ as defined in (1.1). Then, a.s., for any $d \geq 2$,

$$
\begin{gathered}
\liminf _{n \rightarrow \infty} \frac{\left(\lambda^{-1} \log n\right)^{1-1 / \alpha} d_{n}}{\log _{2} n} \geq \frac{d-1}{\alpha \lambda}, \\
\limsup _{n \rightarrow \infty} \frac{\left(\lambda^{-1} \log n\right)^{1-1 / \alpha} d_{n}}{\log _{2} n} \leq \frac{d}{\alpha \lambda} .
\end{gathered}
$$

Note that it follows from Theorem 1.2 that the opposite inequality holds as well in (1.7). Thus, the inequality in (1.7) can be replaced by an equality.

\section{Supporting results and proofs of Theorems 1.1 and $\mathbf{1 . 2}$}

In what follows, $C, C_{1}, C_{2}, C^{\prime}, c_{1}$, etc. will denote constants whose values might change from place to place. For any $x \in \mathbb{R}^{d}$, let $B(x, r)$ denote the open ball of radius $r$ centered at $x$. Let

$$
I(x, r)=\int_{B(x, r)} f(y) \mathrm{d} y .
$$

For $\rho>0$, define $I(\rho, r)=I(\rho e, r)$, where $e$ is the $d$-dimensional unit vector $(1,0,0, \ldots, 0)$. Due to the radial symmetry of $f, I(x, r)=I(\|x\|, r)$. The following lemma, which provides a large $\rho$ asymptotic for $I(\rho, r)$, will be crucial in subsequent calculations. 
Lemma 2.1. Let $\geq 2$, and let $\left\{\rho_{n}\right\}_{n \geq 1}$ and $\left\{r_{n}\right\}_{n \geq 1}$ be sequences of positive numbers satisfying $\rho_{n} \rightarrow \infty, r_{n} / \rho_{n} \rightarrow 0, r_{n}^{2} \rho_{n}^{\alpha-2} \rightarrow 0$, and $r_{n} \rho_{n}^{\alpha-1} \rightarrow \infty$. Then

$$
K_{d} \mathrm{e}^{-\lambda w_{1}(n)} \frac{\left(\Gamma((d+1) / 2)+E_{n}\right)}{\Gamma((d+1) / 2)} g\left(\rho_{n}, r_{n}\right) \leq I\left(\rho_{n}, r_{n}\right) \leq K_{d} \mathrm{e}^{-\lambda w_{2}(n)} g\left(\rho_{n}, r_{n}\right),
$$

where

$$
\begin{aligned}
K_{d} & =A_{d} \theta_{d-1} 2^{(d-1) / 2} \Gamma\left(\frac{d+1}{2}\right), \\
w_{1}(n) & = \begin{cases}\frac{\alpha}{2} r_{n}^{2}\left(\rho_{n}^{2}-2 r_{n} \rho_{n}\right)^{\alpha / 2-1}, & \alpha \leq 2, \\
\frac{\alpha}{2} r_{n}^{2}\left(\rho_{n}^{2}+2 r_{n} \rho_{n}\right)^{\alpha / 2-1}\left[1+(\alpha-2) \rho_{n}^{2}\left(\rho_{n}^{2}-2 r_{n} \rho_{n}\right)^{-1}\right], & \alpha>2,\end{cases} \\
w_{2}(n) & = \begin{cases}\frac{\alpha(\alpha-2)}{2}\left(r_{n} \rho_{n}\right)^{2}\left(\rho_{n}^{2}-2 r_{n} \rho_{n}\right)^{\alpha / 2-2}, & 0<\alpha \leq 2, \\
0, & \alpha>2,\end{cases} \\
\left|E_{n}\right| & \leq \frac{C_{1}}{r_{n} \rho_{n}^{\alpha-1},} \\
g(\rho, r) & =r^{d \mathrm{e}^{-\lambda\left(\rho^{\alpha}-\alpha r \rho^{\alpha-1}\right)}\left(\lambda \alpha r \rho^{\alpha-1}\right)^{-(d+1) / 2},} \quad r, \rho \geq 0,
\end{aligned}
$$

$A_{d}$ is as defined in (1.2), $\theta_{d-1}$ is the volume of the $(d-1)$-dimensional unit ball, and $C_{1}$ is some constant. As $n \rightarrow \infty, E_{n} \rightarrow 0$, and $w_{i}(n) \rightarrow 0, i=1,2$.

The proof of Lemma 2.1 is given in Appendix A. We first prove a Poissonized version of Theorem 1.1 for the number of isolated nodes, i.e. (1.5) with $W_{n}\left(r_{n}\right)$ replaced by $W_{n}^{\prime}\left(r_{n}\right)$. To this end, we first find a sequence $r_{n}$ for which $\mathrm{E}\left[W_{n}^{\prime}\left(r_{n}\right)\right]$ converges. From the Palm theory for Poisson processes (see Equation (8.45) of Penrose (2003)), we obtain

$$
\mathrm{E}\left[W_{n}^{\prime}\left(r_{n}\right)\right]=n \int_{R^{d}} \exp \left(-n I\left(x, r_{n}\right)\right) f(x) \mathrm{d} x .
$$

Changing to polar coordinates gives

$$
\mathrm{E}\left[W_{n}^{\prime}\left(r_{n}\right)\right]=n \int_{0}^{\infty} \exp \left(-n I\left(s, r_{n}\right)\right) f_{R}(s) \mathrm{d} s,
$$

where $f_{R}$ is defined in (1.3). Define the sequence of functions $\left\{\rho_{n}\right\}_{n \geq 1}$ by

$$
\rho_{n}(t)^{\alpha}=\frac{t+a_{n}}{\lambda}, \quad t \geq-a_{n},
$$

where

$$
a_{n}=\log n+\left(\frac{d}{\alpha}-1\right) \log _{2} n-\log \left(\Gamma\left(\frac{d}{\alpha}\right)\right) .
$$

Choose $r_{n}$ so that the remaining factor in (2.5) also converges. Making the change of variable $t=\rho_{n}^{-1}(s)$ in $(2.5)$ we obtain

$$
\mathrm{E}\left[W_{n}^{\prime}\left(r_{n}\right)\right]=\int_{-a_{n}}^{\infty} \exp \left(-n I\left(\rho_{n}(t), r_{n}\right)\right) g_{n}(t) \mathrm{d} t,
$$


where

$$
\begin{aligned}
g_{n}(t) & =n f_{R}\left(\rho_{n}(t)\right) \rho_{n}^{\prime}(t) \\
& =\frac{n \lambda^{d / \alpha-1}}{\Gamma(d / \alpha)}\left(\frac{t+a_{n}}{\lambda}\right)^{d / \alpha-1} \mathrm{e}^{-\left(t+a_{n}\right)} \\
& =\left(\frac{t+a_{n}}{\log n}\right)^{d / \alpha-1} \mathrm{e}^{-t} \\
& =\left(\frac{t+\log n+(d / \alpha-1) \log _{2} n-\log (\Gamma(d / \alpha))}{\log n}\right)^{d / \alpha-1} \mathrm{e}^{-t} \\
& \rightarrow \mathrm{e}^{-t \quad \text { as } n \rightarrow \infty \text { for all } t \in \mathbb{R} .}
\end{aligned}
$$

Lemma 2.2. Suppose that the sequence $\left\{r_{n}\right\}_{n \geq 1}$ satisfies (1.4). Let $t \in \mathbb{R}$, and set $\rho_{n}(t)^{\alpha}=$ $\left(\left(t+a_{n}\right) / \lambda\right) \mathbf{1}_{\left\{t \geq-a_{n}\right\}}$, where $a_{n}$ is as defined in (2.7). Then

$$
\lim _{n \rightarrow \infty} n I\left(\rho_{n}(t), r_{n}\right)=C_{d} \mathrm{e}^{\beta-t},
$$

where $C_{d}$ is as defined in (1.6).

Proof. It is easy to verify that, for each fixed $t \in \mathbb{R}, \rho_{n}=\rho_{n}(t)$ and $r_{n}$ satisfy the conditions of Lemma 2.1, and so we have $n I\left(\rho_{n}, r_{n}\right) \sim K_{d} n g\left(\rho_{n}, r_{n}\right)$. Substituting for $\lambda \rho_{n}^{\alpha}(t)$ from (2.6) and (2.7), we obtain

$$
n I\left(\rho_{n}, r_{n}\right) \sim \frac{n K_{d} \Gamma(d / \alpha) \mathrm{e}^{-t}}{n(\log n)^{d / \alpha-1}} r_{n}^{d} \exp \left(\lambda \alpha r_{n} \rho_{n}^{\alpha-1}\right)\left(\lambda \alpha r_{n} \rho_{n}^{\alpha-1}\right)^{-(d+1) / 2} .
$$

From (1.4) we can write

$$
\begin{aligned}
r_{n} & =\frac{d-1}{\lambda \alpha} \frac{\log _{2} n}{\left(\lambda^{-1} \log n\right)^{1-1 / \alpha}}-\frac{d-1}{2 \lambda \alpha} \frac{\log _{3} n}{\left(\lambda^{-1} \log n\right)^{1-1 / \alpha}}+\frac{\beta+o(1)}{\lambda \alpha\left(\lambda^{-1} \log n\right)^{1-1 / \alpha}} \\
& =\frac{d-1}{\lambda \alpha} \frac{\log _{2} n}{\left(\lambda^{-1} \log n\right)^{1-1 / \alpha}}(1+o(1)),
\end{aligned}
$$

and, hence,

$$
\begin{aligned}
\lambda \alpha r_{n} \rho_{n}^{\alpha-1}= & \left(\frac{(d-1) \log _{2} n}{\left(\lambda^{-1} \log n\right)^{1-1 / \alpha}}-\frac{d-1}{2} \frac{\log _{3} n}{\left(\lambda^{-1} \log n\right)^{1-1 / \alpha}}+\frac{\beta+o(1)}{\left(\lambda^{-1} \log n\right)^{1-1 / \alpha}}\right) \\
& \times\left(\frac{1}{\lambda}\left(t+\log n+\left(\frac{d}{\alpha}-1\right) \log _{2} n-\log \left(\Gamma\left(\frac{d}{\alpha}\right)\right)\right)\right)^{(\alpha-1) / \alpha} \\
= & \left((d-1) \log _{2} n-\frac{d-1}{2} \log _{3} n+\beta+o(1)\right) \\
& \times\left(1+\frac{t}{\log n}+\left(\frac{d}{\alpha}-1\right) \frac{\log _{2} n}{\log n}-\frac{\log (\Gamma(d / \alpha))}{\log n}\right)^{(\alpha-1) / \alpha} \\
= & (d-1) \log _{2} n-\frac{d-1}{2} \log _{3} n+\beta+o(1) .
\end{aligned}
$$


Substituting (2.12) and (2.14) into (2.11), we obtain

$$
\begin{aligned}
n I\left(\rho_{n}, r_{n}\right) \sim & \frac{K_{d} \Gamma(d / \alpha) \mathrm{e}^{-t}}{(\log n)^{d / \alpha-1}}\left(\frac{d-1}{\lambda \alpha} \frac{\log _{2} n}{\left(\lambda^{-1} \log n\right)^{1-1 / \alpha}}(1+o(1))\right)^{d} \\
& \times\left(\frac{\exp \left((d-1) \log _{2} n-\log _{3} n(d-1) / 2+\beta+o(1)\right)}{\left((d-1) \log _{2} n-\log _{3} n(d-1) / 2+\beta+o(1)\right)^{(d+1) / 2}}\right) \\
\rightarrow & C_{d} \mathrm{e}^{\beta-t},
\end{aligned}
$$

where $C_{d}=K_{d} \Gamma(d / \alpha)(d-1)^{(d-1) / 2} \lambda^{-d / \alpha} \alpha^{-d}$. Substituting for $K_{d}$ from (2.1) (and for $A_{d}$ from (1.2)) and simplifying, we obtain

$$
C_{d}=\alpha^{1-d} \theta_{d-1} 2^{(d-1) / 2}(d-1)^{(d-1) / 2} \frac{\Gamma((d+1) / 2) \Gamma(d / 2+1)}{d \pi^{d / 2}} .
$$

Since $\Gamma((d+1) / 2) \Gamma(d / 2+1)=2^{-d} \Gamma(d+1) \sqrt{\pi}$, we obtain the expression for $C_{d}$ given in (1.6).

Lemma 2.3. For any $t \in \mathbb{R}$ and sufficiently large $n$, let $g_{n}(t)$ be as defined in (2.9). There exists a constant $M$ depending on $\alpha, d$, and $\lambda$ such that the following inequalities hold for all large enough $n$.

1. Suppose that $d / \alpha \geq 1$ and $\lambda r_{n}^{\alpha}-a_{n} \leq t \leq 0$ or that $d / \alpha<1$ and $-\log n / \log _{2} n \leq$ $t \leq 0$. Then $g_{n}(t) \leq M \mathrm{e}^{-t}$.

2. For $d / \alpha<1$ and $\lambda r_{n}^{\alpha}-a_{n} \leq t \leq-\log n / \log _{2} n, g_{n}(t) \leq M\left(\log _{2} n / \log n\right)^{d-\alpha} \mathrm{e}^{-t}$.

Remark 2.1. From (2.7), it follows that, for large $n$,

$$
\frac{1}{2} \log n \leq a_{n} \leq 2 \log n
$$

Hence, from (2.12) and (2.16), we have, for large $n, \lambda r_{n}^{\alpha}-a_{n}<-\log n / \log _{2} n$. Thus, the first part of Lemma 2.3 includes the cases $d / \alpha \geq 1$ and $-\log n / \log _{2} n \leq t \leq 0$.

Proof of Lemma 2.3. For the case where $d / \alpha \geq 1$ and $\lambda r_{n}^{\alpha}-a_{n} \leq t \leq 0$, we have, by (2.16),

$$
g_{n}(t) \leq\left(\frac{0+a_{n}}{\log n}\right)^{d / \alpha-1} \mathrm{e}^{-t} \leq 2^{d / \alpha-1} \mathrm{e}^{-t} .
$$

Again using (2.16), if $d / \alpha<1$ and $-\log n / \log _{2} n \leq t \leq 0$, then

$$
\begin{aligned}
g_{n}(t) & \leq\left(\frac{-\log n / \log _{2} n+\log n / 2}{\log n}\right)^{d / \alpha-1} \mathrm{e}^{-t} \\
& \leq\left(\frac{-\log n / 4+\log n / 2}{\log n}\right)^{d / \alpha-1} \mathrm{e}^{-t} \\
& \leq 4^{1-d / \alpha} \mathrm{e}^{-t} .
\end{aligned}
$$

From (2.12), it follows that, for large $n, \lambda r_{n}^{\alpha} \geq\left((d-1) \log _{2} n / 2 \alpha(\log n)^{1-1 / \alpha}\right)^{\alpha}$. Thus, if $d / \alpha<1$ and $\lambda r_{n}^{\alpha}-a_{n} \leq t \leq-\log n / \log _{2} n$,

$$
g_{n}(t) \leq\left(\frac{\lambda r_{n}^{\alpha}-a_{n}+a_{n}}{\log n}\right)^{d / \alpha-1} \mathrm{e}^{-t} \leq\left(\frac{(d-1) \log _{2} n}{2 \alpha \log n}\right)^{d-\alpha} \mathrm{e}^{-t}
$$


Proposition 2.1. Let the sequence $\left\{r_{n}\right\}_{n \geq 1}$ satisfy (1.4), and let $C_{d}$ be as defined in (1.6). Then

$$
\lim _{n \rightarrow \infty} \mathrm{E}\left[W_{n}^{\prime}\right]=\frac{\mathrm{e}^{-\beta}}{C_{d}} .
$$

Proof. From Lemma 2.2 and (2.9), for each $t \in \mathbb{R}$, we have

$$
\lim _{n \rightarrow \infty} \exp \left(-n I\left(\rho_{n}(t), r_{n}\right)\right) g_{n}(t)=\exp \left(-C_{d} \mathrm{e}^{\beta-t}\right) \mathrm{e}^{-t} .
$$

Suppose that we can find integrable bounds for $\exp \left(-n I\left(\rho_{n}(t), r_{n}\right)\right) g_{n}(t)$ that hold for all large $n$. Then, from (2.8), (2.17), and the dominated convergence theorem, we have

$$
\begin{aligned}
\lim _{n \rightarrow \infty} \mathrm{E}\left[W_{n}^{\prime}\left(r_{n}\right)\right] & =\lim _{n \rightarrow \infty} \int_{-a_{n}}^{\infty} \exp \left(-n I\left(\rho_{n}(t), r_{n}\right)\right) g_{n}(t) \mathrm{d} t \\
& =\int_{-\infty}^{\infty} \exp \left(-C_{d} \mathrm{e}^{\beta-t}\right) \mathrm{e}^{-t} \mathrm{~d} t \\
& =\frac{\mathrm{e}^{-\beta}}{C_{d}}
\end{aligned}
$$

We find integrable bounds for $\exp \left(-n I\left(\rho_{n}(t), r_{n}\right)\right) g_{n}(t)$ by dividing the range of $t$ into four parts.

Part 1. First consider $t \geq 0$. For large $n$, since $0.5 \log n<a_{n}<2 \log n$, we have

$$
g_{n}(t) \leq \begin{cases}\left(\frac{t+2 \log n}{\log n}\right)^{d / \alpha-1} \mathrm{e}^{-t} \leq \mathrm{e}^{-t} 2^{d / \alpha} \max (t, 1)^{d / \alpha-1}, & d / \alpha>1, \\ 2^{1-d / \alpha} \mathrm{e}^{-t}, & d / \alpha \leq 1 .\end{cases}
$$

By the above bound on $g_{n}(t)$, it follows that

$$
\exp \left(-n I\left(\rho_{n}(t), r_{n}\right)\right) g_{n}(t) \leq g_{n}(t)
$$

is integrable over $[0, \infty)$.

Part 2. Now consider the range $-\log n / \log _{2} n \leq t \leq 0$. As $\lambda \rho_{n}(t)^{\alpha}=t+a_{n}$, from (2.13) we obtain

$$
\begin{aligned}
\lambda \alpha r_{n} \rho_{n}(t)^{\alpha-1}= & \left((d-1) \log _{2} n-\frac{d-1}{2} \log _{3} n+\beta+o(1)\right) \\
& \times\left(1+\frac{\alpha-1}{\alpha}\left(\frac{t+(d / \alpha-1) \log _{2} n-\log (\Gamma(d / \alpha))}{\log n}\right)\left(1+\zeta_{n}(t)\right)^{-1 / \alpha}\right),
\end{aligned}
$$

where $\left|\zeta_{n}(t)\right| \leq\left|t+(d / \alpha-1) \log _{2} n-\log (\Gamma(d / \alpha))\right|(\log n)^{-1}$. Hence,

$$
\zeta_{n}(t) \mathbf{1}_{\left[-\log n / \log _{2} n, 0\right]}(t) \rightarrow 0
$$

uniformly as $n \rightarrow \infty$. Since $-1 \leq t \log _{2} n / \log n \leq 0$ in the above range of $t$, we can find constants $c_{1}$ and $c_{2}$ such that, for large $n$,

$$
(d-1) \log _{2} n-\frac{d-1}{2} \log _{3} n-c_{1} \leq \lambda \alpha r_{n} \rho_{n}(t)^{\alpha-1} \leq(d-1) \log _{2} n-\frac{d-1}{2} \log _{3} n+c_{2} .
$$


Hence, for all large $n$, we have

$$
\exp \left(\lambda \alpha r_{n} \rho_{n}^{\alpha-1}\right) \geq \frac{(\log n)^{d-1}}{\left(\log _{2} n\right)^{(d-1) / 2}} \mathrm{e}^{-c_{1}}
$$

From Lemma 2.1,

$$
\begin{aligned}
n I\left(\rho_{n}, r_{n}\right) \geq & C_{1} n\left(\Gamma\left(\frac{d+1}{2}\right)+E_{n}\right) \mathrm{e}^{-\lambda w_{1}(n)} g\left(\rho_{n}, r_{n}\right) \\
= & C_{1} n\left(\Gamma\left(\frac{d+1}{2}\right)+E_{n}\right) r_{n}^{d} \frac{\Gamma(d / \alpha) \mathrm{e}^{-t}}{n(\log n)^{d / \alpha-1}} \mathrm{e}^{-\lambda w_{1}(n)} \\
& \times \exp \left(\lambda \alpha r_{n} \rho_{n}^{\alpha-1}\right)\left(\lambda \alpha r_{n} \rho_{n}^{\alpha-1}\right)^{-(d+1) / 2},
\end{aligned}
$$

where $C_{1}=A_{d} \theta_{d-1} 2^{(d-1) / 2}$. Substituting (2.12), (2.20), and (2.21) into the above expression we obtain, for some constant $C$ and large $n$,

$$
\begin{aligned}
n I\left(\rho_{n}, r_{n}\right) \geq & A_{d} \theta_{d-1} 2^{(d-1) / 2}\left(\Gamma\left(\frac{d+1}{2}\right)+E_{n}\right) \mathrm{e}^{-\lambda w_{1}(n)} \\
& \times\left(\frac{(d-1) \log _{2} n}{\lambda \alpha(\lambda-1) \log n)^{1-1 / \alpha}}(1+o(1))\right)^{d} \frac{\Gamma(d / \alpha) \mathrm{e}^{-t}}{(\log n)^{d / \alpha-1}} \frac{(\log n)^{d-1}}{\left(\log _{2} n\right)^{(d-1) / 2}} \\
& \times \mathrm{e}^{-c_{1}}\left((d-1) \log _{2} n-\frac{d-1}{2} \log _{3} n+c_{2}\right)^{-(d+1) / 2} \\
\geq & C\left(\Gamma\left(\frac{d+1}{2}\right)+E_{n}\right) \mathrm{e}^{-\lambda w_{1}(n)} \mathrm{e}^{-t}
\end{aligned}
$$

Suppose that we show, as $n \rightarrow \infty, r_{n} / \rho_{n}(t)$ and $r_{n}^{2} \rho_{n}(t)^{\alpha-2}$ converge uniformly to 0 , and that $r_{n} \rho_{n}(t)^{\alpha-1} \rightarrow \infty$ uniformly for $-\log n / \log _{2} n \leq t \leq 0$. It then follows from (2.2) and (2.3) that $w_{1}(n)$ and $E_{n}$ converge uniformly to 0 . Hence, we can find a constant $c^{\prime}>0$ such that

$$
n I\left(\rho_{n}, r_{n}\right) \geq c^{\prime} \mathrm{e}^{-t}
$$

From the above inequality and part 1 of Lemma 2.3 (see also Remark 2.1), there exists a constant $c$ such that, for all large $n$, we have

$$
\exp \left(-n I\left(\rho_{n}(t), r_{n}\right)\right) g_{n}(t) \leq c \exp \left(-c^{\prime} \mathrm{e}^{-t}\right) \mathrm{e}^{-t}, \quad-\frac{\log n}{\log _{2} n} \leq t \leq 0 .
$$

This upper bound is integrable over $t \in(-\infty, 0)$. We now verify the three conditions assumed above. That $r_{n} \rho_{n}(t)^{\alpha-1} \rightarrow \infty$ uniformly follows from (2.20). From (2.12), for some constant $c_{1}$ and large $n$, we have $r_{n} \leq c_{1} \log _{2} n /(\log n)^{1-1 / \alpha}$. Since $-\log n / \log _{2} n \leq t \leq 0$, by (2.6), for all sufficiently large $n$,

$$
\lambda \rho_{n}(t)^{\alpha} \geq \log n-\frac{\log n}{\log _{2} n}+\left(\frac{d}{\alpha}-1\right) \log _{2} n-\log \left(\Gamma\left(\frac{d}{\alpha}\right)\right) \geq \frac{1}{2} \log n .
$$

So, for large $n$, we can find a constant $c_{2}$ such that $r_{n} / \rho_{n}(t) \leq c_{2} \log _{2} n / \log n \rightarrow 0$ as $n \rightarrow \infty$. Next we show that $r_{n}^{2} \rho_{n}(t)^{\alpha-2} \rightarrow 0$ as $n \rightarrow \infty$ uniformly over $-\log n / \log _{2} n \leq t \leq 0$. By 
(1.4) and (2.6), we obtain

$$
\begin{aligned}
(\lambda \alpha)^{2} r_{n}^{2} \rho_{n}(t)^{\alpha-2}= & \left(\frac{(d-1) \log _{2} n}{\left(\lambda^{-1} \log n\right)^{1-1 / \alpha}}-\frac{d-1}{2} \frac{\log _{3} n}{\left(\lambda^{-1} \log n\right)^{1-1 / \alpha}}+\frac{\beta+o(1)}{\left(\lambda^{-1} \log n\right)^{1-1 / \alpha}}\right)^{2} \\
& \times\left(\frac{1}{\lambda}\left(t+\log n+\left(\frac{d}{\alpha}-1\right) \log _{2} n-\log \left(\Gamma\left(\frac{d}{\alpha}\right)\right)\right)\right)^{(\alpha-2) / \alpha} \\
= & \lambda\left(\frac{\left((d-1) \log _{2} n-\log _{3} n(d-1) / 2+\beta+o(1)\right)^{2}}{\log n}\right) \\
& \times\left(1+\frac{t+(d / \alpha-1) \log _{2} n-\log (\Gamma(d / \alpha))}{\log n}\right)^{(\alpha-2) / \alpha} .
\end{aligned}
$$

Since $-\log n / \log _{2} n \leq t \leq 0$, the right-hand side of the above equation is bounded by

$$
\begin{aligned}
& \lambda \frac{\left((d-1) \log _{2} n-\log _{3} n(d-1) / 2+\beta+o(1)\right)^{2}}{\log n} \\
& \times\left(1+\frac{(d / \alpha-1) \log _{2} n-\log (\Gamma(d / \alpha))}{\log n}\right)^{(\alpha-2) / \alpha}
\end{aligned}
$$

for $\alpha \geq 2$, and by

$$
\begin{aligned}
& \lambda \frac{\left((d-1) \log _{2} n-\log _{3} n(d-1) / 2+\beta+o(1)\right)^{2}}{\log n} \\
& \times\left(1-\frac{\log n / \log _{2} n-(d / \alpha-1) \log _{2} n+\log (\Gamma(d / \alpha))}{\log n}\right)^{(\alpha-2) / \alpha}
\end{aligned}
$$

for $0<\alpha<2$. Both these bounds are independent of $t$ and converge to 0 as $n \rightarrow \infty$.

Part 3. Next, consider the range $\lambda r_{n}^{\alpha}-a_{n} \leq t \leq-\log n / \log _{2} n$ (see Remark 2.1). From the first inequality we have $r_{n} \leq \rho_{n}(t)$, and, hence,

$$
\begin{aligned}
I\left(\rho_{n}(t), r_{n}\right) & =\int_{B\left(\rho_{n}(t) e, r_{n}\right)} A_{d} \mathrm{e}^{-\lambda\|x\|^{\alpha}} \mathrm{d} x \\
& >\int_{B\left(\rho_{n}(t) e, r_{n}\right),\|x\| \leq \rho_{n}(t)} A_{d} \mathrm{e}^{-\lambda\|x\|^{\alpha}} \mathrm{d} x \\
& \geq A_{d} \mathrm{e}^{-\lambda \rho_{n}(t)^{\alpha}}\left|B\left(\rho_{n}(t) e, r_{n}\right) \cap B\left(0, \rho_{n}(t)\right)\right|,
\end{aligned}
$$

where $|\cdot|$ denotes the volume and $e=(1,0, \ldots, 0) \in \mathbb{R}^{d}$. Inscribe a sphere of diameter $r_{n}$ inside $B\left(\rho_{n}(t) e, r_{n}\right) \cap B\left(0, \rho_{n}(t)\right)$ (see Figure 1). Hence,

$$
\left|B\left(\rho_{n}(t) e, r_{n}\right) \cap B\left(0, \rho_{n}(t)\right)\right| \geq \frac{\theta_{d} r_{n}^{d}}{2^{d}} .
$$

Substituting (2.6), (2.12), and (2.27) into (2.26), we have, for large $n$,

$$
\begin{aligned}
I\left(\rho_{n}(t), r_{n}\right) & \geq c^{\prime \prime} \mathrm{e}^{-\lambda \rho_{n}(t)^{\alpha}} r_{n}^{d} \\
& =\frac{c^{\prime \prime \prime} \mathrm{e}^{-t}}{n(\log n)^{d / \alpha-1}} \frac{\left(\log _{2} n\right)^{d}}{(\log n)^{d-d / \alpha}}\left(1-\frac{\log _{3} n}{2 \log _{2} n}+\frac{\beta+o(1)}{(d-1) \log _{2} n}\right)^{d} \\
& \geq c^{*} n^{-1}(\log n)^{1-d}\left(\log _{2} n\right)^{d} \mathrm{e}^{-t} \\
& =q_{n} \mathrm{e}^{-t}
\end{aligned}
$$




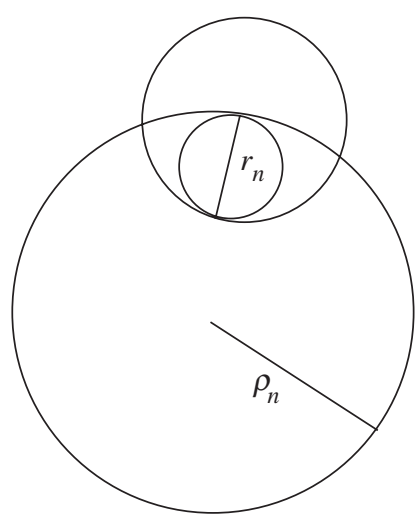

Figure 1.

where

$$
q_{n}=c^{*}(\log n)^{1-d}\left(\log _{2} n\right)^{d} n^{-1} .
$$

From Lemma 2.3 and (2.28), we obtain,

$$
\begin{aligned}
& \int_{\lambda r_{n}^{\alpha}-a_{n}}^{-\log n / \log _{2} n} \exp \left(-n I\left(\rho_{n}(t), r_{n}\right)\right) g_{n}(t) \mathrm{d} t \\
& \leq \begin{cases}M \int_{\lambda r_{n}^{\alpha}-a_{n}}^{-\log n / \log _{2} n} \exp \left(-n q_{n} \mathrm{e}^{-t}\right) \mathrm{e}^{-t} \mathrm{~d} t, & d / \alpha \geq 1, \\
M\left(\frac{\log _{2} n}{\log n}\right)^{d-\alpha} \int_{\lambda r_{n}^{\alpha}-a_{n}}^{-\log n / \log _{2} n} \exp \left(-n q_{n} \mathrm{e}^{-t}\right) \mathrm{e}^{-t} \mathrm{~d} t, & d / \alpha<1,\end{cases} \\
& \leq \begin{cases}M \int_{\exp \left(\log n / \log _{2} n\right)}^{\exp \left(a_{n}-\lambda r_{n}^{\alpha}\right)} \mathrm{e}^{-n q_{n} y} \mathrm{~d} y, & d / \alpha \geq 1, \\
M\left(\frac{\log _{2} n}{\log n}\right)^{d-\alpha} \int_{\exp \left(\log n / \log _{2} n\right)}^{\exp \left(a_{n}-\lambda r_{n}^{\alpha}\right)} \mathrm{e}^{-n q_{n} y} \mathrm{~d} y, & d / \alpha<1,\end{cases} \\
& \leq \begin{cases}\frac{M}{n q_{n}} \exp \left(-n q_{n} \mathrm{e}^{\log n / \log _{2} n}\right), & d / \alpha \geq 1, \\
\frac{M}{n q_{n}}\left(\frac{\log _{2} n}{\log n}\right)^{d-\alpha} \exp \left(-n q_{n} \mathrm{e}^{\log n / \log _{2} n}\right), & d / \alpha<1 .\end{cases}
\end{aligned}
$$

We have

$$
\begin{aligned}
\frac{M}{n q_{n}} \exp \left(-n q_{n} \mathrm{e}^{\log n / \log _{2} n}\right) & =\frac{M}{n q_{n}} \exp \left(-n^{1+1 / \log _{2} n} q_{n}\right) \\
& =C \frac{(\log n)^{d-1}}{\left(\log _{2} n\right)^{d}} \exp \left(-c^{*} n^{1 / \log _{2} n}(\log n)^{1-d}\left(\log _{2} n\right)^{d}\right)
\end{aligned}
$$


Consider the exponent $c^{*} n^{1 / \log _{2} n}(\log n)^{1-d}\left(\log _{2} n\right)^{d}$. Taking logarithms we obtain, for large $n$,

$$
\log \left(c^{*}\right)+\frac{\log n}{\log _{2} n}+(1-d) \log _{2} n+d \log _{3} n \geq \frac{\log n}{2 \log _{2} n} .
$$

Hence,

$$
c^{*} n^{1 / \log _{2} n}(\log n)^{1-d}\left(\log _{2} n\right)^{d} \geq \mathrm{e}^{\log n / 2 \log _{2} n} .
$$

Using (2.32) in (2.31), we obtain

$$
\frac{M}{n q_{n}} \exp \left(-n q_{n} \mathrm{e}^{\log n / \log _{2} n}\right) \leq C\left(\frac{\log n}{\log _{2} n}\right)^{d-1} \frac{1}{\log _{2} n} \exp \left(-\mathrm{e}^{\log n / 2 \log _{2} n}\right) \rightarrow 0,
$$

since the exponent is decaying exponentially fast in $\log n / \log _{2} n$. Using the inequality from (2.33) in (2.30) for the case $d / \alpha<1$, we obtain

$$
\frac{M}{n q_{n}}\left(\frac{\log _{2} n}{\log n}\right)^{d-\alpha} \exp \left(-n q_{n} \mathrm{e}^{\log n / \log _{2} n}\right) \leq \frac{C(\log n)^{\alpha-1}}{\left(\log _{2} n\right)^{\alpha}} \exp \left(-\mathrm{e}^{\log n / 2 \log _{2} n}\right),
$$

which converges to 0 as $n \rightarrow \infty$, by the same argument as above. From (2.30), (2.33), and (2.34), we have

$$
\int_{\lambda r_{n}^{\alpha}-a_{n}}^{-\log n / \log _{2} n} \exp \left(-n I\left(\rho_{n}(t), r_{n}\right)\right) g_{n}(t) \mathrm{d} t \rightarrow 0 .
$$

Part 4. Finally, consider the case $-a_{n} \leq t \leq \lambda r_{n}^{\alpha}-a_{n}$. The second inequality implies that $r_{n} \geq \rho_{n}(t)$. Hence, for large $n$, we have

$$
n I\left(\rho_{n}(t), r_{n}\right)=n \int_{B\left(\rho_{n}(t) e, r_{n}\right)} A_{d} \mathrm{e}^{-\lambda\|x\|^{\alpha}} \mathrm{d} x \geq n \int_{B\left(r_{n} e, r_{n}\right)} A_{d} \mathrm{e}^{-\lambda\|x\|^{\alpha}} \mathrm{d} x \geq c_{1} n \mathrm{e}^{-\lambda\left(2 r_{n}\right)^{\alpha}} r_{n}^{d} .
$$

For large $n$ from (2.12), we have

$$
\frac{(d-1) \log _{2} n}{2 \lambda^{1 / \alpha} \alpha(\log n)^{1-1 / \alpha}} \leq r_{n} \leq \frac{2(d-1) \log _{2} n}{\lambda^{1 / \alpha} \alpha(\log n)^{1-1 / \alpha}} .
$$

Fix $0<\varepsilon_{1}, \varepsilon_{2}<1$ such that $\varepsilon=\varepsilon_{1}+\varepsilon_{2}<1$. Substituting (2.37) into (2.36) we obtain, for large $n$ and some positive constants $c_{2}$ and $c_{3}$,

$$
\begin{aligned}
n I\left(\rho_{n}(t), r_{n}\right) & \geq c_{2} n \exp \left(-c_{3} \frac{\left(\log _{2} n\right)^{\alpha}}{(\log n)^{\alpha-1}}\right) \frac{\left(\log _{2} n\right)^{d}}{(\log n)^{d-d / \alpha}} \\
& \geq c_{2} n^{1-\varepsilon_{1}} \exp \left(-c_{3}\left(\frac{\log _{2} n}{\log n}\right)^{\alpha} \log n\right) \\
& =c_{2} n^{1-\varepsilon_{1}-c_{3}\left(\log _{2} n / \log n\right)^{\alpha}} \\
& \geq c_{2} n^{1-\varepsilon_{1}-\varepsilon_{2}} \\
& =c_{2} n^{1-\varepsilon} .
\end{aligned}
$$


From (2.9), (2.38), and the fact that, for large $n, a_{n}<2 \log n$, we obtain

$$
\begin{aligned}
\int_{-a_{n}}^{\lambda r_{n}^{\alpha}-a_{n}} \mathrm{e}^{-n I\left(\rho_{n}(t), r_{n}\right)} g_{n}(t) \mathrm{d} t & \leq \frac{\mathrm{e}^{-c_{2} n^{1-\varepsilon}}}{(\log n)^{d / \alpha-1}} \int_{-a_{n}}^{\lambda r_{n}^{\alpha}-a_{n}}\left(t+a_{n}\right)^{d / \alpha-1} \mathrm{e}^{-t} \mathrm{~d} t \\
& \leq \frac{\mathrm{e}^{a_{n}} \mathrm{e}^{-c_{2} n^{1-\varepsilon}}}{(\log n)^{d / \alpha-1}} \int_{0}^{\infty} u^{d / \alpha-1} \mathrm{e}^{-u} \mathrm{~d} u \\
& \leq \frac{c n^{2} \mathrm{e}^{-c_{2} n^{1-\varepsilon}}}{(\log n)^{d / \alpha-1}} \\
& \rightarrow 0
\end{aligned}
$$

This completes the proof of Proposition 2.1.

Theorem 2.1. Let $\alpha \in \mathbb{R}$, and let $r_{n}$ be as defined in (1.4). Then

$$
W_{n}^{\prime}\left(r_{n}\right) \stackrel{\mathcal{D}}{\rightarrow} \operatorname{Po}\left(\mathrm{e}^{-\beta} / C_{d}\right),
$$

where $C_{d}$ is as defined in (1.6) and $\operatorname{Po}\left(\mathrm{e}^{-\beta} / C_{d}\right)$ is the Poisson random variable with mean $\mathrm{e}^{-\beta} / C_{d}$.

Proof. From Theorem 6.7 of Penrose (2003) and Proposition 2.1, the total variation distance, $\mathrm{d}_{\mathrm{TV}}\left(W_{n}^{\prime}\left(r_{n}\right), \operatorname{Po}\left(\mathrm{E}\left[W_{n}^{\prime}\left(r_{n}\right)\right]\right)\right)$ is bounded by a constant times $J_{1}(n)+J_{2}(n)$, where $J_{1}(n)$ and $J_{2}(n)$ are defined as

$$
\begin{aligned}
& J_{1}(n)=n^{2} \int_{\mathbb{R}^{d}} \exp \left(-n I\left(x, r_{n}\right)\right) f(x) \mathrm{d} x \int_{B\left(x, 3 r_{n}\right)} \exp \left(-n I\left(y, r_{n}\right)\right) f(y) \mathrm{d} y, \\
& J_{2}(n)=n^{2} \int_{\mathbb{R}^{d}} f(x) \mathrm{d} x \int_{B\left(x, 3 r_{n}\right) \backslash B\left(x, r_{n}\right)} \exp \left(-n I^{(2)}\left(x, y, r_{n}\right)\right) f(y) \mathrm{d} y,
\end{aligned}
$$

where $I^{(2)}(x, y, r)=\int_{B(x, r) \cup B(y, r)} f(z) \mathrm{d} z$. Theorem 2.1 follows from Proposition 2.1 if we show that $J_{i}(n) \rightarrow 0$ as $n \rightarrow \infty, i=1,2$. We first analyze $J_{1}$. Let $\rho_{n}(t)$ and $g_{n}(t)$ be as defined in Lemma 2.2 and (2.9), respectively. We have

$$
J_{1}(n)=n^{2} \int_{-a_{n}}^{\infty} \exp \left(-n I\left(\rho_{n}(t), r_{n}\right)\right) g_{n}(t) \mathrm{d} t \int_{B\left(\rho_{n}(t) e, 3 r_{n}\right)} \exp \left(-n I\left(y, r_{n}\right)\right) f(y) \mathrm{d} y .
$$

Write $J_{1}(n)=J_{11}(n)+J_{12}(n)$, where

$$
\begin{aligned}
J_{11}(n)= & \int_{-a_{n}}^{-\log n / \log _{2} n} \exp \left(-n I\left(\rho_{n}(t), r_{n}\right)\right) g_{n}(t) \mathrm{d} t \\
& \times \int_{B\left(\rho_{n}(t) e, 3 r_{n}\right)} \exp \left(-n I\left(y, r_{n}\right)\right) n f(y) \mathrm{d} y \\
J_{12}(n)= & \int_{-\log n / \log _{2} n}^{\infty} \exp \left(-n I\left(\rho_{n}(t), r_{n}\right)\right) g_{n}(t) \mathrm{d} t \\
& \times \int_{B\left(\rho_{n}(t) e, 3 r_{n}\right)} \exp \left(-n I\left(y, r_{n}\right)\right) n f(y) \mathrm{d} y .
\end{aligned}
$$


From (2.8) and Proposition 2.1, the inner integral in $J_{11}$,

$$
\begin{aligned}
\int_{B\left(\rho_{n}(t) e, 3 r_{n}\right)} \exp \left(-n I\left(y, r_{n}\right)\right) n f(y) \mathrm{d} y & \leq \int_{-a_{n}}^{\infty} \exp \left(-n I\left(\rho_{n}\left(t^{\prime}\right), r_{n}\right)\right) g_{n}\left(t^{\prime}\right) \mathrm{d} t^{\prime} \\
& =\mathrm{E}\left[W_{n}^{\prime}\left(r_{n}\right)\right] \\
& \rightarrow \frac{\mathrm{e}^{-\beta}}{C_{d}}
\end{aligned}
$$

as $n \rightarrow \infty$. Thus, for any $\varepsilon>0$ and all large $n$, we have

$$
J_{11}(n) \leq(1+\varepsilon) \frac{\mathrm{e}^{-\beta}}{C_{d}} \int_{-a_{n}}^{-\log n / \log _{2} n} \exp \left(-n I\left(\rho_{n}(t), r_{n}\right)\right) g_{n}(t) \mathrm{d} t .
$$

It follows from (2.35) and (2.39) that $J_{11}(n) \rightarrow 0$. Next we will show that $J_{12}(n) \rightarrow 0$ as $n \rightarrow \infty$. Define $B_{n}(t)=\left\{t^{\prime}: \rho_{n}(t)-3 r_{n} \leq \rho_{n}\left(t^{\prime}\right) \leq \rho_{n}(t)+3 r_{n}\right\}$. Note that, for $t \geq$ $-\log n / \log _{2} n, \rho_{n}(t)-3 r_{n} \geq 0$. The inner integral in $J_{12}(n)$ reduces to

$$
\begin{aligned}
\int_{B\left(\rho_{n}(t) e, 3 r_{n}\right)} \exp \left(-n I\left(y, r_{n}\right)\right) n f(y) \mathrm{d} y \\
\leq\left(2 \sin ^{-1}\left(\frac{3 r_{n}}{\rho_{n}(t)}\right)\right)^{d-1} \int_{B_{n}(t)} \exp \left(-n I\left(\rho_{n}\left(t^{\prime}\right), r_{n}\right)\right) g_{n}\left(t^{\prime}\right) \mathrm{d} t^{\prime} \\
\leq\left(2 \sin ^{-1}\left(\frac{3 r_{n}}{\rho_{n}(t)}\right)\right)^{d-1} \int_{-a_{n}}^{\infty} \exp \left(-n I\left(\rho_{n}\left(t^{\prime}\right), r_{n}\right)\right) g_{n}\left(t^{\prime}\right) \mathrm{d} t^{\prime} \\
\leq 2^{d-1}(1+\varepsilon) \frac{\mathrm{e}^{-\beta}}{C_{d}}\left(\sin ^{-1}\left(\frac{3 r_{n}}{\rho_{n}(t)}\right)\right)^{d-1} \\
\leq C\left(\frac{\log _{2} n}{\log n}\right)^{d-1},
\end{aligned}
$$

since, for all large $n$ and $t \in\left(-\log n / \log _{2} n, \infty\right)$, we can find constants $c, c^{\prime}$, and $\varepsilon>0$ such that $0 \leq 3 r_{n} / \rho_{n}(t) \leq c \log _{2} n / \log n \rightarrow 0$, and $\sin ^{-1}(x) \leq c^{\prime} x$ for all $x \in[0, \varepsilon]$. Thus, the inner integral in $J_{12}$ converges uniformly to 0 as $n \rightarrow \infty$. Hence, $J_{12}$ converges to 0 from the last statement and the fact that the bounds in (2.19) and (2.25) are integrable over $[0, \infty)$ and $(-\infty, 0]$, respectively.

We now show that $J_{2}$ as defined in (2.40) converges to 0 . Write $J_{2}(n)=\sum_{k=1}^{3} J_{2 k}(n)$, where

$$
J_{2 k}(n)=n^{2} \int_{\mathbb{R}^{d}} f(x) \mathrm{d} x \int_{A_{k}(n)} \exp \left(-n I^{(2)}\left(x, y, r_{n}\right)\right) f(y) \mathrm{d} y, \quad k=1,2,3,
$$

with $A_{1}(n)=\left\{2 r_{n} \leq\|x-y\| \leq 3 r_{n}\right\}, A_{2}(n)=\left\{r_{n} \leq\|x-y\| \leq 2 r_{n},\|x\| \leq\|y\|\right\}$, and $A_{3}(n)=\left\{r_{n} \leq\|x-y\| \leq 2 r_{n},\|y\| \leq\|x\|\right\}$. Since on $A_{1}(n), I^{(2)}\left(x, y, r_{n}\right)=$ $I\left(x, r_{n}\right)+I\left(y, r_{n}\right)$, we obtain

$$
\begin{aligned}
J_{21}(n) & =n^{2} \int_{\mathbb{R}^{d}} \exp \left(-n I\left(x, r_{n}\right)\right) f(x) \mathrm{d} x \int_{\left\{y: 2 r_{n} \leq\|x-y\| \leq 3 r_{n}\right\}} \exp \left(-n I\left(y, r_{n}\right)\right) f(y) \mathrm{d} y \\
& \leq n^{2} \int_{\mathbb{R}^{d}} \exp \left(-n I\left(x, r_{n}\right)\right) f(x) \mathrm{d} x \int_{B\left(x, 3 r_{n}\right)} \exp \left(-n I\left(y, r_{n}\right)\right) f(y) \mathrm{d} y \\
& =J_{1}(n),
\end{aligned}
$$




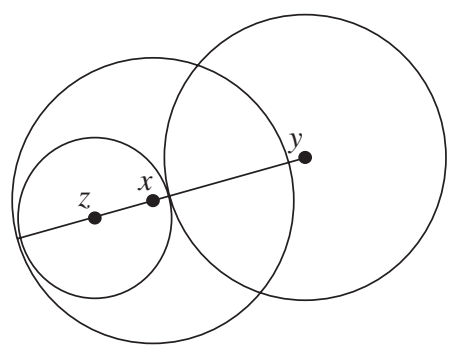

FIGURE 2.

which has already been shown to converge to 0 . Next we analyze $J_{22}(n)$ as $n \rightarrow \infty$. The proof for $J_{23}(n)$ is the same and so we omit it.

Let $B\left(z(x, y), \rho_{1}\right)$ be the ball with center $z=z(x, y)$ (see Figure 2) and radius $\rho_{1}=$ $\rho_{1}(x, y) \geq r_{n} / 2$ inscribed inside $B\left(x, r_{n}\right) \backslash B\left(y, r_{n}\right)$. Then

$$
\begin{aligned}
I^{(2)}\left(x, y, r_{n}\right) & \geq I\left(z(x, y), \rho_{1}\right)+I\left(y, r_{n}\right) \\
& \geq I\left(z(x, y), r_{n} / 2\right)+I\left(y, r_{n}\right) \\
& \geq I\left(x, r_{n} / 2\right)+I\left(y, r_{n}\right),
\end{aligned}
$$

where the last inequality follows since $\|z\|<\|x\|$. Thus,

$$
\begin{aligned}
J_{22}(n) & \leq n^{2} \int_{\mathbb{R}^{d}} \exp \left(-n I\left(x, \frac{r_{n}}{2}\right)\right) f(x) \mathrm{d} x \int_{A_{2}(n)} \exp \left(-n I\left(y, r_{n}\right)\right) f(y) \mathrm{d} y \\
& \leq n^{2} \int_{\mathbb{R}^{d}} \exp \left(-n I\left(x, \frac{r_{n}}{2}\right)\right) f(x) \mathrm{d} x \int_{B\left(x, 3 r_{n}\right)} \exp \left(-n I\left(y, r_{n}\right)\right) f(y) \mathrm{d} y .
\end{aligned}
$$

Write $J_{22}(n)=J_{1}^{*}(n)+J_{2}^{*}(n)+J_{3}^{*}(n)$, where

$$
J_{i}^{*}(n)=\int_{D_{i}} \exp \left(-n I\left(\rho_{n}(t), \frac{r_{n}}{2}\right)\right) g_{n}(t) \mathrm{d} t \int_{B\left(\rho_{n}(t) e, 3 r_{n}\right)} \exp \left(-n I\left(y, r_{n}\right)\right) n f(y) \mathrm{d} y
$$

for $i=1,2,3$, where $D_{1}=\left[-a_{n},-\log n / \log _{2} n\right), D_{2}=\left[-\log n / \log _{2} n, 0\right)$, and $D_{3}=$ $[0, \infty)$. The proof of $J_{i}^{*} \rightarrow 0$ as $n \rightarrow \infty$ for $i=1,3$ proceeds in exactly the same manner as in the cases of $J_{11}$ and $J_{12}$ by replacing $r_{n}$ by $r_{n} / 2$ while estimating the outer integrals. In the case of $J_{2}^{*}$, we proceed exactly as in the case of $J_{12}$ (see (2.41)) to obtain

$$
J_{2}^{*}(n) \leq C\left(\frac{\log _{2} n}{\log n}\right)^{d-1} \int_{D_{2}} \exp \left(-n I\left(\rho_{n}(t), \frac{r_{n}}{2}\right)\right) g_{n}(t) \mathrm{d} t .
$$

We will show that there exists a constant $C_{1}$ such that, for large $n$,

$$
\int_{D_{2}} \exp \left(-n I\left(\rho_{n}(t), \frac{r_{n}}{2}\right)\right) g_{n}(t) \mathrm{d} t \leq C_{1} \frac{(\log n)^{(d-1) / 2}}{\left(\log _{2} n\right)^{(d-1) / 4}} .
$$

Substituting (2.43) into (2.42) we obtain

$$
J_{2}^{*}(n) \leq C^{\prime}\left(\frac{\log _{2} n}{\log n}\right)^{d-1} \frac{(\log n)^{(d-1) / 2}}{\left(\log _{2} n\right)^{(d-1) / 4}} \rightarrow 0 \quad \text { as } n \rightarrow \infty .
$$


This completes the proof of Theorem 2.1 once we show (2.43). We first obtain a bound for the integrand in (2.43) as in (2.25) by replacing $r_{n}$ by $r_{n} / 2$. Using Lemma 2.1 (see also (2.22)), we can find a constant $c_{0}>0$ such that

$$
\begin{aligned}
n I\left(\rho_{n}(t), \frac{r_{n}}{2}\right) \geq & c_{0} n\left(\Gamma\left(\frac{d+1}{2}\right)+E_{n}\right) r_{n}^{d} \mathrm{e}^{-\lambda w_{1}(n)}\left(r_{n} \rho_{n}^{\alpha-1}\right)^{-(d+1) / 2} \\
& \times \exp \left(-\lambda\left(\rho_{n}^{\alpha}-\alpha r_{n} \rho_{n}^{\alpha-1}\right)\right) \exp \left(-\frac{\lambda \alpha}{2} r_{n} \rho_{n}^{\alpha-1}\right),
\end{aligned}
$$

where the functions $E_{n}$ and $w_{1}(n)$ are as in Lemma 2.1, but with $r_{n}$ replaced by $r_{n} / 2$. It is easy to see that the conditions for the uniform convergence to 0 of $E_{n}$ and $w_{1}(n)$ that were verified below (2.25) for $r_{n}$ hold for $r_{n} / 2$ as well. Hence, the computations leading to (2.24) can be used to estimate the right-hand side of (2.44) leaving out the last factor. This gives, for large $n$ and some constant $c^{\prime \prime}>0$,

$$
n I\left(\rho_{n}(t), \frac{r_{n}}{2}\right) \geq c^{\prime \prime} \mathrm{e}^{-t} \exp \left(-\frac{\lambda \alpha}{2} r_{n} \rho_{n}(t)^{\alpha-1}\right) .
$$

From (2.20) we have $\exp \left(-\lambda \alpha r_{n} \rho_{n}(t)^{\alpha-1} / 2\right) \geq\left(\log _{2} n\right)^{(d-1) / 4}(\log n)^{-(d-1) / 2} \mathrm{e}^{-c_{2} / 2}$. Therefore,

$$
n I\left(\rho_{n}(t), \frac{r_{n}}{2}\right) \geq c_{3} \frac{\left(\log _{2} n\right)^{(d-1) / 4}}{(\log n)^{(d-1) / 2}} \mathrm{e}^{-t} .
$$

From (2.45) and part 1 of Lemma 2.3, we obtain

$$
\exp \left(-n I\left(\rho_{n}(t), \frac{r_{n}}{2}\right)\right) g_{n}(t) \leq M \exp \left(-c_{3} \frac{\left(\log _{2} n\right)^{(d-1) / 4}}{(\log n)^{(d-1) / 2}} \mathrm{e}^{-t}\right) \mathrm{e}^{-t}
$$

for all large $n$. Hence, for all large $n$, we have

$$
\begin{aligned}
\int_{D_{2}} \exp \left(-n I\left(\rho_{n}(t), \frac{r_{n}}{2}\right)\right) g_{n}(t) \mathrm{d} t & \leq M \int_{-\infty}^{0} \exp \left(-c_{3} \frac{\left(\log _{2} n\right)^{(d-1) / 4}}{(\log n)^{(d-1) / 2}} \mathrm{e}^{-t}\right) \mathrm{e}^{-t} \mathrm{~d} t \\
& \leq \frac{M}{c_{3}} \frac{(\log n)^{(d-1) / 2}}{\left(\log _{2} n\right)^{(d-1) / 4}} .
\end{aligned}
$$

This proves (2.43).

Proof of Theorem 1.1. For each positive integer $n$, set $m_{1}(n)=n-n^{3 / 4}$ and $m_{2}(n)=$ $n+n^{3 / 4}$. Recall that the Poisson sequence $N_{n}$ is assumed to be nondecreasing. Let $r_{n}$ be as in the statement of the theorem. Since $m_{i}(n) \sim n$, it is easy to see that Proposition 2.1 and Theorem 2.1 hold with $n$ replaced by $m_{i}(n)$, that is,

$$
\mathrm{E}\left[W_{m_{i}(n)}^{\prime}\left(r_{n}\right)\right] \rightarrow \frac{\mathrm{e}^{-\beta}}{C_{d}}, \quad W_{m_{i}(n)}^{\prime}\left(r_{n}\right) \stackrel{\mathscr{D}}{\rightarrow} \operatorname{Po}\left(\frac{\mathrm{e}^{-\beta}}{C_{d}}\right), \quad i=1,2 .
$$

Let $\mathcal{P}_{n}^{-}=\mathcal{P}_{m_{1}(n)}$ and $\mathcal{P}_{n}^{+}=\mathcal{P}_{m_{2}(n)}$. Let $A^{\mathrm{c}}$ denote the complement of set $A$. Let $H_{n}=\left\{\mathcal{P}_{n}^{-} \subseteq\right.$ $\left.\mathcal{X}_{n} \subseteq \mathcal{P}_{n}^{+}\right\}$. Let $A_{n}$ be the event that there exists a point $Y \in \mathcal{P}_{n}^{+} \backslash \mathcal{P}_{n}^{-}$such that $Y$ is isolated in $G\left(\mathcal{P}_{n}^{-} \cup\{Y\}, r_{n}\right)$. Let $B_{n}$ be the event that one or more points of $\mathcal{P}_{n}^{+} \backslash \mathcal{P}_{n}^{-}$lie within distance $r_{n}$ of a point $X$ of $\mathcal{P}_{n}^{-}$with degree 0 in $G\left(\mathcal{P}_{n}^{-}, r_{n}\right)$. Then $\left\{W_{n}\left(r_{n}\right) \neq W_{n}^{\prime}\left(r_{n}\right)\right\} \subseteq A_{n} \cup B_{n} \cup H_{n}^{\mathrm{c}}$. Thus, the proof is complete if we show that $\mathrm{P}\left[A_{n}\right], \mathrm{P}\left[B_{n}\right], \mathrm{P}\left[H_{n}^{\mathrm{c}}\right]$ all converge to 0 . We have

$$
\mathrm{P}\left[H_{n}^{\mathrm{c}}\right] \leq \mathrm{P}\left[\left|N_{m_{1}(n)}-m_{1}(n)\right| \geq n^{3 / 4}\right]+\mathrm{P}\left[\left|N_{m_{2}(n)}-m_{2}(n)\right| \geq n^{3 / 4}\right] \rightarrow 0
$$

as $n \rightarrow \infty$ by Chebyshev's inequality. 
Let $Y \in \mathbb{R}^{d}$ be a point distributed according to the density $f$ independent of $\mathcal{P}_{n}^{-}$. By the Palm theory we have

$$
\mathrm{P}\left[A_{n}\right] \leq 2 n^{3 / 4} \mathrm{P}\left[Y \text { is isolated in } G\left(\mathcal{P}_{n}^{-} \cup\{Y\}, r_{n}\right)\right]=2 n^{3 / 4} m_{1}(n)^{-1} \mathrm{E}\left[W_{m_{1}(n)}^{\prime}\left(r_{n}\right)\right],
$$

which converges to 0 as $n \rightarrow \infty$. By Boole's inequality and the Palm theory,

$$
\begin{aligned}
\mathrm{P}\left[B_{n}\right] & \leq 2 n^{3 / 4} \mathrm{P}\left[\text { there is a isolated point of } G\left(\mathcal{P}_{n}^{-}, r_{n}\right) \text { in } B\left(Y, r_{n}\right)\right] \\
& \leq 2 n^{7 / 4} \int_{\mathbb{R}^{d}} f(y) \mathrm{d} y \int_{B\left(y, r_{n}\right)} \exp \left(-m_{1}(n) I\left(x, r_{n}\right)\right) f(x) \mathrm{d} x .
\end{aligned}
$$

By interchanging the order of integration, we obtain

$$
\begin{aligned}
\mathrm{P}\left[B_{n}\right] & \leq 2 n^{7 / 4} \int_{\mathbb{R}^{d}} I\left(x, r_{n}\right) \exp \left(-m_{1}(n) I\left(x, r_{n}\right)\right) f(x) \mathrm{d} x \\
& =2 n^{3 / 4} \int_{-a_{n}}^{\infty} I\left(\rho_{n}(t), r_{n}\right) \exp \left(-m_{1}(n) I\left(\rho_{n}(t), r_{n}\right)\right) g_{n}(t) \mathrm{d} t .
\end{aligned}
$$

From (2.9) and (2.10), we obtain

$$
2 n^{3 / 4} I\left(\rho_{n}(t), r_{n}\right) \exp \left(-m_{1}(n) I\left(\rho_{n}(t), r_{n}\right)\right) g_{n}(t) \rightarrow 0 .
$$

Thus, the integrand in (2.46) converges pointwise to 0 as $n \rightarrow \infty$. To complete the proof, we need to find integrable bounds for the left-hand side of the above equation. Let $0<\varepsilon<1$ be fixed. For large $n$, we have $m_{1}(n) \geq(1-\varepsilon) n$. Hence, for large $n$,

$$
\begin{aligned}
n^{3 / 4} I & \left(\rho_{n}(t), r_{n}\right) \exp \left(-m_{1}(n) I\left(\rho_{n}(t), r_{n}\right)\right) g_{n}(t) \\
& \leq n^{3 / 4} I\left(\rho_{n}(t), r_{n}\right) \exp \left(-(1-\varepsilon) n I\left(\rho_{n}(t), r_{n}\right)\right) g_{n}(t) .
\end{aligned}
$$

Thus, it suffices to find integrable bounds for the right-hand side expression in (2.47). The procedure for doing this is the same as in the proof of Proposition 2.1, where we obtained integrable bounds for $\exp \left(-n I\left(\rho_{n}(t), r_{n}\right)\right) g_{n}(t)$ by dividing the range of the integral $\left[-a_{n}, \infty\right)$ into four parts, and in each part finding a lower bound for $n I\left(\rho_{n}(t), r_{n}\right)$ of the form $c_{i} h_{i}(n, t)$ and upper bounds for $g_{n}(t), i=1, \ldots, 4$. We can use the same bounds for the factor $\exp (-(1-$ $\left.\varepsilon) n I\left(\rho_{n}(t), r_{n}\right)\right) g_{n}(t)$ by replacing the constants $c_{i}$ by $(1-\varepsilon) c_{i}, i=1, \ldots, 4$ (see below). Thus, for each of the four domains analyzed in Proposition 2.1, we need to find an upper bound for $n^{3 / 4} I\left(\rho_{n}(t), r_{n}\right)$, and then verify that the product of this bound and the one obtained for $\exp \left(-(1-\varepsilon) n I\left(\rho_{n}(t), r_{n}\right)\right) g_{n}(t)$ is integrable.

Part 1. Let $t \geq 0$. From (2.15) we obtain, for large $n$,

$$
n^{3 / 4} I\left(\rho_{n}(t), r_{n}\right) \leq n^{3 / 4} I\left(\rho_{n}(0), r_{n}\right) \leq(1+\varepsilon) C_{d} \mathrm{e}^{\beta} n^{-1 / 4} .
$$

Hence, for large $n$,

$$
n^{3 / 4} I\left(\rho_{n}(t), r_{n}\right) \exp \left(-(1-\varepsilon) n I\left(\rho_{n}(t), r_{n}\right)\right) g_{n}(t) \leq(1+\varepsilon) C_{d} \mathrm{e}^{\beta} n^{-1 / 4} g_{n}(t),
$$

which is integrable over $[0, \infty)$ by (2.18) for each $n$, and converges to 0 as $n \rightarrow \infty$.

Part 2. Next suppose that $-\log n / \log _{2} n \leq t \leq 0$. Using Lemma 2.1 and proceeding as in (2.22) and (2.23), with $w_{1}(n)$ replaced by $w_{2}(n)$ and $E_{n}$ replaced by 0 , we obtain, for large $n$ and some constant $c^{\prime \prime}$,

$$
n^{3 / 4} I\left(\rho_{n}(t), r_{n}\right) \leq n^{-1 / 4} c^{\prime \prime} \mathrm{e}^{-t} .
$$


This together with (2.24) and part 1 of Lemma 2.3 yields

$$
n^{3 / 4} I\left(\rho_{n}(t), r_{n}\right) \exp \left(-(1-\varepsilon) n I\left(\rho_{n}(t), r_{n}\right)\right) g_{n}(t) \leq c_{1} n^{-1 / 4} \exp \left(-(1-\varepsilon) c^{\prime} \mathrm{e}^{-t}\right) \mathrm{e}^{-2 t}
$$

for large $n$ and some constant $c_{1}$. This bound is integrable in $t$ over the interval $(-\infty, 0)$, and converges to 0 as $n \rightarrow \infty$.

Part 3. Next, consider the range $\lambda r_{n}^{\alpha}-a_{n} \leq t \leq-\log n / \log _{2} n$. For large $n$, we have

$$
I\left(\rho_{n}(t), r_{n}\right) \leq \int_{B\left(0, r_{n}\right)} A_{d} \mathrm{e}^{-\lambda\|x\|^{\alpha}} \mathrm{d} x \leq A_{d} r_{n}^{d} \leq c_{2} \frac{\left(\log _{2} n\right)^{d}}{(\log n)^{d-d / \alpha}},
$$

where the last inequality in (2.48) follows from (2.37). Note that the above bound is independent of $t$. Hence, for large $n$,

$$
\int_{\lambda r_{n}^{\alpha}-a_{n}}^{-\log n / \log _{2} n} n^{3 / 4} I\left(\rho_{n}(t), r_{n}\right) \exp \left(-(1-\varepsilon) n I\left(\rho_{n}(t), r_{n}\right)\right) g_{n}(t) \mathrm{d} t
$$

will be bounded by $n^{3 / 4}$ times the last expression in (2.48) times the bound obtained in (2.30), with the constant $c^{*}$ (see (2.29)) replaced by $(1-\varepsilon) c^{*}$. The bounds obtained in (2.30) are in turn bounded above in (2.33) or (2.34), depending on whether $d / \alpha \geq 1$ or $d / \alpha \leq 1$. If $d / \alpha \geq 1$ then, for large $n$, the product of the bounds in (2.33) and (2.48) will be less than a constant times

$$
n^{3 / 4}(\log n)^{d / \alpha-1} \exp \left(-\mathrm{e}^{\log n / 2 \log _{2} n}\right) \leq n^{3 / 4}(\log n)^{d / \alpha-1} \exp \left(-\mathrm{e}^{\log _{2} n}\right)=n^{-1 / 4}(\log n)^{d / \alpha-1},
$$

which converges to 0 as $n \rightarrow \infty$. The same reasoning applies to the case $d / \alpha<1$ by using (2.34) instead of (2.33).

Part 4. Finally, consider the case $-a_{n} \leq t \leq \lambda r_{n}^{\alpha}-a_{n}$. Using (2.39) and (2.48) with $c_{2}$ replaced by $(1-\varepsilon) c_{2}$, we obtain, for large $n$ and some constant $c$,

$$
\begin{gathered}
\int_{-a_{n}}^{\lambda r_{n}^{\alpha}-a_{n}} n^{3 / 4} I\left(\rho_{n}(t), r_{n}\right) \exp \left(-(1-\varepsilon) n I\left(\rho_{n}(t), r_{n}\right)\right) g_{n}(t) \mathrm{d} t \\
\quad \leq \frac{c n^{3}\left(\log _{2} n\right)^{d} \exp \left(-(1-\varepsilon) c_{2} n^{1-\varepsilon}\right)}{(\log n)^{d-1}},
\end{gathered}
$$

which converges to 0 as $n \rightarrow \infty$.

Proof of Theorem 1.2. Define a sequence $\left\{r_{n}\right\}_{n \geq 1}$ by

$$
\lambda \alpha\left(\lambda^{-1} \log n\right)^{1-1 / \alpha} r_{n}-(d-1) \log _{2} n+\frac{d-1}{2} \log _{3} n=\beta .
$$

Then by Theorem 1.1 we have

$$
\lim _{n \rightarrow \infty} \mathrm{P}\left[d_{n} \leq r_{n}\right]=\lim _{n \rightarrow \infty} \mathrm{P}\left[W_{n}\left(r_{n}\right)=0\right]=\exp \left(-\frac{\mathrm{e}^{-\beta}}{C_{d}}\right) .
$$

The result now follows by taking $\beta=\gamma-\log \left(C_{d}\right)$. 


\section{Proof of Theorem 1.3}

In order to prove strong laws for the LNND for graphs with densities having compact support, we cover the support of the density using an appropriate collection of concentric balls and then show summability of certain events involving the distribution of the points of $\mathcal{X}_{n}$ on these balls. The results then follow by an application of the Borel-Cantelli lemma. In the case of densities having unbounded support, the region to be covered changes with $n$ and must be determined first. The following lemma gives us the regions of interest when the points in $\mathcal{X}_{n}=\left\{X_{1}, X_{2}, \ldots, X_{n}\right\}, n \geq 1$, are distributed according to the probability density function $f$ given by (1.1).

For any set $A$, let $A^{\mathrm{c}}$ denote its complement. For any two real sequences $\left\{a_{n}\right\}_{n \geq 1}$ and $\left\{b_{n}\right\}_{n \geq 1}, a_{n} \gtrsim b_{n}$ means that $a_{n} \geq c_{n}, n \geq 1$, for some sequence $\left\{c_{n}\right\}_{n \geq 1}$ with $c_{n} \sim b_{n}$.

For any $c \in \mathbb{R}$ and large enough $n$, define

$$
R_{n}(c)=\left(\frac{1}{\lambda}\left(\log n+\frac{c+d-\alpha}{\alpha} \log _{2} n\right)\right)^{1 / \alpha} .
$$

Define the events $U_{n}(c)=\left\{\mathcal{X}_{n} \subset B\left(0, R_{n}(c)\right)\right\}$, and, for any $c<0$, let $V_{n}(c)=\left\{X_{n} \cap\right.$ $\left.\left(B\left(0, R_{n}(0)\right) \backslash B\left(0, R_{n}(c)\right)\right) \neq \varnothing\right\}$ for all large enough $n$ for which $R_{n}(c)>0$, and arbitrarily otherwise.

Lemma 3.1. Let the events $U_{n}(c)$ and $V_{n}(c), n \geq 1$, be as defined above. Then

1. $\mathrm{P}\left[U_{n}^{\mathrm{c}}(c)\right.$ infinitely often $]=0$ for any $c>\alpha$, and

2. $\mathrm{P}\left[V_{n}^{\mathrm{c}}(c)\right.$ infinitely often $]=0$ for any $c<0$.

The above results are also true with $\mathcal{X}_{n}$ replaced by $\mathcal{P}_{\lambda_{n}}$ provided that $\lambda_{n} \sim n$.

Thus, for almost all realizations of the sequence $\left\{\mathcal{X}_{n}\right\}_{n \geq 1}$, all points of $\mathcal{X}_{n}$ will lie within the ball $B\left(0, R_{n}(c)\right)$ for any $c>\alpha$ eventually, and, for any $c<0$, there will be at least one point of $\mathcal{X}_{n}$ in $B\left(0, R_{n}(0)\right) \backslash B\left(0, R_{n}(c)\right)$ eventually.

Proof of Lemma 3.1. From (1.3) we obtain

$$
\int_{\tilde{R}}^{\infty} f_{R}(r) \mathrm{d} r \sim A_{d}^{\prime}(\lambda \alpha)^{-1} \tilde{R}^{d-\alpha} \mathrm{e}^{-\lambda \tilde{R}^{\alpha}} \text { as } \tilde{R} \rightarrow \infty .
$$

Fix an integer $a>1$, and define the subsequence $\left\{n_{k}\right\}_{k \geq 1}$ by $n_{k}=a^{k}$. For large $k$, we have

$$
\begin{aligned}
\mathrm{P}\left[\bigcup_{n=n_{k}}^{n_{k+1}} U_{n}^{\mathrm{c}}(c)\right] & \leq \mathrm{P}\left[\text { at least one vertex of } \mathcal{X}_{n_{k+1}} \text { is in } B^{\mathrm{c}}\left(0, R_{n_{k}}(c)\right)\right] \\
& \leq n_{k+1} \int_{R_{n_{k}}(c)}^{\infty} f_{R}(r) \mathrm{d} r \\
& \sim A_{d}^{\prime}(\lambda \alpha)^{-1} n_{k+1} R_{n_{k}}^{d-\alpha}(c) \mathrm{e}^{-\lambda R_{n_{k}}^{\alpha}(c)} \\
& \leq \frac{C}{k^{c / \alpha}}
\end{aligned}
$$

Thus, the above probability is summable for $c>\alpha$, and the first part of Lemma 3.1 follows from the Borel-Cantelli lemma. 
Next, suppose that $c<0$ and let $\left\{n_{k}\right\}_{k \geq 1}$ be as above. Note that, for all large $k, R_{n_{k+1}}(c)<$ $R_{n_{k}}(0)$. From (3.1) we obtain, for all large $n$,

$$
\frac{1}{2 \lambda^{(d-\alpha) / \alpha}} \frac{1}{n(\log n)^{b / \alpha}} \leq R_{n}^{d-\alpha}(b) \mathrm{e}^{-\lambda R_{n}^{\alpha}(b)} \leq \frac{2}{\lambda^{(d-\alpha) / \alpha}} \frac{1}{n(\log n)^{b / \alpha}}, \quad b=c, 0 .
$$

Using the inequality $1-x \leq \mathrm{e}^{-x},(3.2)$, and the above inequalities, we obtain, for large $k$,

$$
\begin{aligned}
\mathrm{P}\left[\bigcup_{n=n_{k}}^{n_{k+1}} V_{n}^{\mathrm{c}}(c)\right] & \leq \mathrm{P}\left[\mathcal{X}_{n_{k}} \cap\left(B\left(0, R_{n_{k}}(0)\right) \backslash B\left(0, R_{n_{k+1}}(c)\right)\right)=\varnothing\right] \\
& \leq \exp \left(-n_{k} \int_{R_{n_{k+1}}(c)}^{R_{n_{k}}(0)} A_{d}^{\prime} \mathrm{e}^{-\lambda r^{\alpha}} r^{d-1} \mathrm{~d} r\right) \\
& \leq \exp \left(-n_{k} A_{d}^{\prime}(\lambda \alpha)^{-1}\left(\frac{1}{2} R_{n_{k+1}}^{d-\alpha}(c) \mathrm{e}^{-\lambda R_{n_{k+1}}^{\alpha}(c)}-2 R_{n_{k}}^{d-\alpha}(0) \mathrm{e}^{-\lambda R_{n_{k}}^{\alpha}(0)}\right)\right) \\
& =\exp \left(-\frac{A_{d}^{\prime}(\lambda \alpha)^{-1}}{\lambda^{(d-\alpha) / \alpha}}\left(\frac{1}{4 a(\log a)^{c / \alpha}}(k+1)^{-c / \alpha}-4\right)\right),
\end{aligned}
$$

which is summable for all $c<0$. The second part of Lemma 3.1 now follows from the Borel-Cantelli lemma. If $\mathcal{X}_{n}$ is replaced by $\mathcal{P}_{\lambda_{n}}$, where $\lambda_{n} \sim n$, then

$$
\begin{aligned}
\mathrm{P}\left[U_{n}^{\mathrm{c}}(c)\right] & =1-\exp \left(-\lambda_{n}\left(1-I\left(0, R_{n}(c)\right)\right)\right) \\
& \lesssim \lambda_{n} A_{d}^{\prime}(\lambda \alpha)^{-1} R_{n}^{d-\alpha}(c) \exp \left(-\lambda R_{n}^{\alpha}(c)\right) \\
& \sim n A_{d}^{\prime}(\lambda \alpha)^{-1} R_{n}^{d-\alpha}(c) \exp \left(-\lambda R_{n}^{\alpha}(c)\right),
\end{aligned}
$$

which is the same as the asymptotic behavior of $\mathrm{P}\left[U_{n}^{\mathrm{c}}(c)\right]$ in case of $\mathcal{X}_{n}$. Similarly, we can show that $\mathrm{P}\left[V_{n}^{\mathrm{c}}(c)\right]$ has the same asymptotic behavior as in the case of $\mathcal{X}_{n}$. Thus, the results stated for $\mathcal{X}_{n}$ also hold for $\mathcal{P}_{\lambda_{n}}$.

Proposition 3.1. For any $t>d / \alpha \lambda$, let $r_{n}(t)=t\left(\lambda^{-1} \log n\right)^{1 / \alpha-1} \log _{2} n$. Then, with probability $1, d_{n} \leq r_{n}(t)$ for all large enough $n$.

Proof. Fix $t>d / \alpha \lambda$, and choose $u \in(d / \alpha \lambda, t)$. Pick $c>\alpha$ and $\varepsilon$ satisfying $0<\varepsilon<$ $u /(2+u), \varepsilon+u<t$, and

$$
\frac{c+\alpha(d-1)}{\alpha^{2} \lambda}=\frac{d}{\alpha \lambda}+\frac{c-\alpha}{\alpha^{2} \lambda}<(1-\varepsilon) u<t .
$$

From Lemma 3.1, a.s., $\mathcal{X}_{n} \subset B\left(0, R_{n}(c)\right)$ for all large enough $n$, where $R_{n}(c)$ is as defined in (3.1). For $m=1,2, \ldots$, let $v(m)=a^{m}$ for some integer $a>1$. Define the sequence of functions $\left\{\tilde{r}_{m}(v)\right\}_{m \geq 1}$ by

$$
\tilde{r}_{m}(v)= \begin{cases}r_{v(m+1)}(v) & \text { if } \alpha>1, \\ r_{v(m)}(v) & \text { if } \alpha \leq 1 .\end{cases}
$$

This is required since $r_{n}(u)$ is decreasing in $n$ if $\alpha>1$, and increasing if $\alpha \leq 1$. Let $\kappa_{m}$ (the covering number) be the minimum number of balls of radii $\tilde{r}_{m}(\varepsilon)$ required to cover the ball $B\left(0, R_{v(m+1)}(c)\right)$. Since

$$
\frac{r_{v(m+1)}(\varepsilon)}{r_{v(m)}(\varepsilon)} \rightarrow 1 \quad \text { as } m \rightarrow \infty
$$


we can find constants $C, C_{1}$, and $C_{2}$ such that, for all sufficiently large $m$,

$$
\begin{aligned}
\kappa_{m} & \leq C \frac{R_{\nu(m+1)}(c)^{d}}{\tilde{r}_{m}(\varepsilon)^{d}} \\
& \leq C_{1} \frac{R_{v(m+1)}(c)^{d}}{r_{v(m+1)}(\varepsilon)^{d}} \\
& =\frac{C_{1}}{\lambda^{d} \varepsilon^{d}} \frac{\left(\log (v(m+1))+\log _{2}(v(m+1))(c+d-\alpha) / \alpha\right)^{d / \alpha}}{(\log (v(m+1)))^{d / \alpha-d}\left(\log _{2}(v(m+1))\right)^{d}} \\
& \leq C_{2}\left(\frac{m+1}{\log (m+1)}\right)^{d} .
\end{aligned}
$$

Consider a deterministic set $\left\{x_{1}^{m}, \ldots, x_{\kappa_{m}}^{m}\right\} \subset B\left(0, R_{v(m+1)}(c)\right)$, such that

$$
B\left(0, R_{\nu(m+1)}(c)\right) \subset \bigcup_{i=1}^{\kappa_{m}} B\left(x_{i}^{m}, \tilde{r}_{m}(\varepsilon)\right) .
$$

Given $x \in \mathbb{R}^{d}$, define $A_{m}(x)$ to be the annulus $B\left(x, \tilde{r}_{m}(u)\right) \backslash B\left(x, \tilde{r}_{m}(\varepsilon)\right)$, and let $F_{m}(x)$ be the event such that no vertex of $\mathcal{X}_{v(m)}$ lies in $A_{m}(x)$, i.e.

$$
F_{m}(x)=\left\{\mathcal{X}_{v(m)}\left[A_{m}(x)\right]=0\right\}
$$

where $\mathcal{X}[B]$ denotes the number of points of the finite set $\mathcal{X}$ that lie in $B$. For any $x \in$ $B\left(0, R_{v(m+1)}(c)\right)$, we have, by the radial symmetry of $f$,

$$
\mathrm{P}\left[X_{i} \in A_{m}(x)\right]=\int_{A_{m}(x)} f(y) \mathrm{d} y=\int_{A_{m}(|x| e)} f(y) \mathrm{d} y,
$$

where $e=(1,0, \ldots, 0) \in \mathbb{R}^{d}$. The aim is to find a lower bound for the above probability. Note that

$$
\frac{r_{n}(u)}{R_{n}(c)}=\frac{u\left(\lambda^{-1} \log n\right)^{1 / \alpha-1} \log _{2} n}{\left(\lambda^{-1}\left(\log n+\log _{2} n(c+d-\alpha) / \alpha\right)\right)^{1 / \alpha}} \rightarrow 0
$$

as $n \rightarrow \infty$. Thus, in (3.7) we integrate the density $f$ over a relatively small annulus $A_{m}(x)$ centered at $x$ which lies in a ball $B\left(0, R_{v(m+1)}(c)\right)$ of relatively larger radius. Since $f$ is radially symmetric and decreasing, it should be possible to obtain a lower bound for $x=R_{v(m+1)}(c) e$. To show this, first consider the case in which $|x|>\tilde{r}_{m}(u)$. Then, $y=\left(y_{1}, \ldots, y_{d}\right) \in A_{m}(|x| e)$ implies that $y_{1}>0$. Combining this with the fact that $|x| \leq R_{v(m+1)}(c)$, we have

$$
\left|y+\left(R_{\nu(m+1)}(c)-|x|\right) e\right|=\left|\left(y_{1}+\left(R_{\nu(m+1)}(c)-|x|\right), y_{2}, \ldots, y_{d}\right)\right| \geq|y| .
$$

On the other hand, if $|x| \leq \tilde{r}_{m}(u)$ then, for any $y \in A_{m}(x),|y| \leq 2 \tilde{r}_{m}(u)$. Hence, for all large $m$, we obtain

$$
\left|y+\left(R_{\nu(m+1)}(c)-|x|\right) e\right| \geq R_{\nu(m+1)}(c)-\tilde{r}_{m}(u)-|y| \geq|y|+\left(R_{\nu(m+1)}(c)-5 \tilde{r}_{m}(u)\right) \geq|y|,
$$


where the last inequality follows using (3.8). From (3.7), (3.9), (3.10), and the fact that the density $f$ is radially symmetric and decreasing, we obtain, for all large $m$,

$$
\begin{aligned}
\mathrm{P}\left[X_{i} \in A_{m}(x)\right] & \geq \int_{A_{m}(|x| e)} f\left(\left(R_{v(m+1)}(c)-|x|\right) e+y\right) \mathrm{d} y \\
& =\int_{A_{m}\left(R_{v(m+1)}(c) e\right)} f(y) \mathrm{d} y \\
& =I\left(R_{v(m+1)}(c), \tilde{r}_{m}(u)\right)-I\left(R_{v(m+1)}(c), \tilde{r}_{m}(\varepsilon)\right) .
\end{aligned}
$$

To apply Lemma 2.1 with $\rho_{n}=R_{n}(c)$ and $r_{n}=r_{n}(v), v=\varepsilon$, $u$, we first check the four conditions of the lemma. As $n \rightarrow \infty$, by definition, $R_{n}^{\alpha}(c) \rightarrow \infty$, and

$$
\begin{aligned}
\frac{r_{n}(v)}{R_{n}(c)} & =\lambda^{1-1 / \alpha} v \log _{2} n / \log n\left(\frac{1}{\lambda}\left(1+\frac{c+d-\alpha}{\alpha} \frac{\log _{2} n}{\log n}\right)\right)^{1 / \alpha} \rightarrow 0, \\
r_{n}^{2}(v) R_{n}^{\alpha-2}(c) & =\frac{v^{2} \lambda^{2-2 / \alpha}\left(\log _{2} n\right)^{2}}{\log n}\left(\frac{1}{\lambda}\left(1+\frac{c+d-\alpha}{\alpha} \frac{\log _{2} n}{\log n}\right)\right)^{1-2 / \alpha} \rightarrow 0, \\
r_{n}(v) R_{n}^{\alpha-1}(c) & =v \lambda^{1-1 / \alpha} \log _{2} n\left(\frac{1}{\lambda}\left(1+\frac{c+d-\alpha}{\alpha} \frac{\log _{2} n}{\log n}\right)\right)^{1-1 / \alpha} \rightarrow \infty
\end{aligned}
$$

The above limits can be easily seen to hold if we take $R_{n}$ and $r_{n}$ to be $R_{v(m+1)}$ and $r_{v(m)}$, respectively, by using (3.4). Hence, by Lemma 2.1 (noting from the last line of the lemma that $w_{i}(n), i=1,2$, and $E_{n}$ converge to 0 ), we can find positive constants $c_{1}$ and $c_{2}$ (depending on u) such that, for large $m$,

$$
c_{1} g\left(R_{v(m+1)}(c), \tilde{r}_{m}(u)\right) \leq(\lambda \alpha)^{-(d+1) / 2} I\left(R_{\nu(m+1)}(c), \tilde{r}_{m}(u)\right) \leq c_{2} g\left(R_{v(m+1)}(c), \tilde{r}_{m}(u)\right) .
$$

Substituting (3.12) and (2.4) into (3.11), we have, for large $m$,

$$
\begin{aligned}
\mathrm{P}\left[X_{i} \in\right. & \left.A_{m}(x)\right] \\
\geq & \exp \left(-\lambda R_{v(m+1)}^{\alpha}(c)\right)\left(R_{v(m+1)}^{\alpha-1}(c)\right)^{-(d+1) / 2} \\
& \times\left(c_{1}(u) \exp \left(\lambda \alpha \tilde{r}_{m}(u) R_{v(m+1)}^{\alpha-1}(c)\right)\left(\tilde{r}_{m}(u)\right)^{(d-1) / 2}\right. \\
& \left.\quad-c_{2}(\varepsilon) \exp \left(\lambda \alpha \tilde{r}_{m}(\varepsilon) R_{v(m+1)}^{\alpha-1}(c)\right)\left(\tilde{r}_{m}(\varepsilon)\right)^{(d-1) / 2}\right) \\
= & q_{m} .
\end{aligned}
$$

We now compute a lower bound for $q_{m}$. For large $n$, we can find a constant $C_{3}$ such that

$$
\begin{aligned}
\exp ( & \left.-\lambda R_{n}^{\alpha}(c)\right)\left(R_{n}^{\alpha-1}(c)\right)^{-(d+1) / 2} \\
& =\frac{1}{n(\log (n))^{(c+d-\alpha) / \alpha}}\left(\frac{1}{\lambda}\left(\log n+\frac{c+d-\alpha}{\alpha} \log _{2} n\right)\right)^{-(1-1 / \alpha)(d+1) / 2} \\
& \geq C_{3} \frac{(\log n)^{-(1-1 / \alpha)(d+1) / 2}}{n(\log n)^{(c+d-\alpha) / \alpha}} .
\end{aligned}
$$

Furthermore,

$$
\lambda \alpha r_{n}(u) R_{n}^{\alpha-1}(c)=\lambda \alpha u \log _{2} n\left(1+\frac{\log _{2} n(c+d-\alpha) / \alpha}{\log n}\right)^{1-1 / \alpha} .
$$


From the above equation (and using (3.4) for the $\alpha \leq 1$ case), we obtain, for large $m$,

$$
(1-\varepsilon) \lambda \alpha u \log _{2} v(m+1) \leq \lambda \alpha \tilde{r}_{m}(u) R_{\nu(m+1)}^{\alpha-1}(c) \leq 2 \lambda \alpha u \log _{2} v(m+1) .
$$

By the above inequality and (3.4), we can find a constant $C_{4}=C_{4}(u)$ such that, for large $m$,

$$
\begin{aligned}
& \exp \left(\lambda \alpha \tilde{r}_{m}(u) R_{v(m+1)}^{\alpha-1}(c)\right)\left(\tilde{r}_{m}(u)\right)^{(d-1) / 2} \\
& \quad \geq C_{4} \frac{\exp \left((1-\varepsilon) \lambda \alpha u \log _{2}(v(m+1))\right)\left(\log _{2} v(m+1)\right)^{(d-1) / 2}}{(\log v(m+1))^{(1-1 / \alpha)(d-1) / 2}} .
\end{aligned}
$$

From (3.16), with $u$ replaced by $\varepsilon$, for some constant $C_{5}=C_{5}(\varepsilon)$ and large $m$, we obtain

$$
\begin{aligned}
& \exp \left(\lambda \alpha \tilde{r}_{m}(\varepsilon) R_{\nu(m+1)}^{\alpha-1}(c)\right)\left(\tilde{r}_{m}(\varepsilon)\right)^{(d-1) / 2} \\
& \quad \leq C_{5} \frac{\exp \left(2 \lambda \alpha \varepsilon \log _{2}(v(m+1))\right)\left(\log _{2}(v(m+1))\right)^{(d-1) / 2}}{(\log (v(m+1)))^{(1-1 / \alpha)(d-1) / 2}} .
\end{aligned}
$$

Substituting (3.14), (3.18), and (3.19) into (3.13), we obtain, for large $m$,

$$
\begin{aligned}
q_{m} \geq & C_{3} \frac{(\log (v(m+1)))^{-(1-1 / \alpha)(d+1) / 2}}{v(m+1)(\log (\nu(m+1)))^{(c+d-\alpha) / \alpha}} \\
& \times \frac{\left(\log _{2}(\nu(m+1))\right)^{(d-1) / 2} \exp \left((1-\varepsilon) \lambda \alpha u \log _{2}(\nu(m+1))\right)}{(\log (\nu(m+1)))^{(1-1 / \alpha)(d-1) / 2}} \\
& \times\left(c_{1} C_{4}-c_{2} C_{5} \exp \left(\lambda \alpha(2 \varepsilon-(1-\varepsilon) u) \log _{2}(\nu(m+1))\right)\right) .
\end{aligned}
$$

Since $\varepsilon<u /(2+u), 2 \varepsilon-(1-\varepsilon) u<0$, and, hence, for large $m$, the term on the last line above is bounded below by $c_{1} C_{4} / 2$. Hence, for large $m$, we have

$$
\begin{aligned}
q_{m} \geq & \frac{C_{3} c_{1} C_{4}}{2} \frac{(\log (v(m+1)))^{-(1-1 / \alpha)(d+1) / 2}}{v(m+1)(\log (v(m+1)))^{(c+d-\alpha) / \alpha}} \\
& \times \frac{\left(\log _{2}(v(m+1))\right)^{(d-1) / 2} \exp \left((1-\varepsilon) \lambda \alpha u \log _{2}(v(m+1))\right)}{(\log (v(m+1)))^{(1-1 / \alpha)(d-1) / 2}} \\
\geq & C \frac{(\log (m+1))^{(d-1) / 2}}{a^{m}(m+1)^{c / \alpha+d-(1-\varepsilon) \lambda \alpha u-1}}
\end{aligned}
$$

for some constant $C$. Hence, for large $m$, from (3.6), (3.13), (3.20), and the inequality $1-x \leq$ $\mathrm{e}^{-x}$, we obtain, for any $x \in B\left(0, R_{\nu(m+1)}(c)\right)$,

$$
\begin{aligned}
\mathrm{P}\left[F_{m}(x)\right] & =\left(1-\mathrm{P}\left[X_{1} \in A_{m}(x)\right]\right)^{v(m)} \\
& \leq\left(1-q_{m}\right)^{v(m)} \\
& \leq \exp \left(-v(m) q_{m}\right) \\
& \leq \exp \left(-C \frac{(\log (m+1))^{(d-1) / 2}}{(m+1)^{c / \alpha+d-(1-\varepsilon) \lambda \alpha u-1}}\right) .
\end{aligned}
$$

Set $G_{m}=\bigcup_{i=1}^{\kappa_{m}} F_{m}\left(x_{i}^{m}\right)$. From (3.5) and (3.21), we have, for large $m$,

$$
\begin{aligned}
\mathrm{P}\left[G_{m}\right] & \leq \sum_{i=1}^{\kappa_{m}} \mathrm{P}\left[F_{m}\left(x_{i}^{m}\right)\right] \\
& \leq C_{2}\left(\frac{m+1}{\log (m+1)}\right)^{d} \exp \left(-C \frac{(\log (m+1))^{(d-1) / 2}}{(m+1)^{\lambda \alpha\left((c+\alpha(d-1)) / \lambda \alpha^{2}-(1-\varepsilon) u\right)}}\right),
\end{aligned}
$$


which is summable in $m$ by (3.3). By the Borel-Cantelli lemma, a.s., $G_{m}$ occurs only for finitely many $m$. Choose $n>a$, and take $m$ such that $a^{m} \leq n \leq a^{m+1}$. If $d_{n}>r_{n}(t)$ then there exists an $X \in \mathcal{X}_{n}$ such that $\mathcal{X}_{n}\left[B\left(X, r_{n}(t)\right) \backslash\{X\}\right]=0$. By Lemma 3.1, $X$ will a.s. be in $B\left(0, R_{v(m+1)}(c)\right)$ for all large enough $m$. Hence, a.s., if $n$ is large enough then there is some $i \leq \kappa_{m}$ such that $X \in B\left(x_{i}^{m}, \tilde{r}_{m}(\varepsilon)\right)$. Since

$$
\tilde{r}_{m}(\varepsilon)+\tilde{r}_{m}(u) \leq \tilde{r}_{m}(t) \leq r_{n}(t),
$$

it follows that $F_{m}\left(x_{i}\right)$ and, hence, $G_{m}$ occur. Since, a.s., $G_{m}$ occurs only finitely often, it follows that $d_{n} \leq r_{n}(t)$ a.s. for all large $n$. This completes the proof of Proposition 3.1.

Proposition 3.2. For any $t \in(0,(d-1) / \alpha \lambda)$, let $r_{n}(t)=t \log _{2} n\left(\lambda^{-1} \log n\right)^{1 / \alpha-1}$. Then, with probability $1, d_{n} \geq r_{n}(t)$ eventually.

We prove Proposition 3.2 via the Poissonization technique, which uses the following lemma (see Lemma 1.4 of Penrose (2003)).

Lemma 3.2. Let $N$ be a Poisson random variable with mean $\lambda$. Then there exist constants $c$ and $\lambda_{1}$ such that, for all $\lambda>\lambda_{1}$,

$$
\max \left\{\mathrm{P}\left[N>\lambda+\frac{1}{2} \lambda^{3 / 4}\right], \mathrm{P}\left[N<\lambda-\frac{1}{2} \lambda^{3 / 4}\right]\right\} \leq c \exp \left(-\frac{1}{9} \sqrt{\lambda}\right),
$$

Proof of Proposition 3.2. Enlarging the probability space, assume that there exists nondecreasing sequences of Poisson variables $\{N(n)\}_{n \geq 1}$ and $\{M(n)\}_{n \geq 1}$ with $\mathrm{E}[N(n)]=n-n^{3 / 4}$ and $\mathrm{E}[M(n)]=2 n^{3 / 4}$, independent of each other and of the sequence $\left\{X_{1}, X_{2}, \ldots\right\}$. Define the point processes

$$
\mathcal{P}_{n}^{-}=\left\{X_{1}, X_{2}, \ldots, X_{N(n)}\right\}, \quad \mathcal{P}_{n}^{+}=\left\{X_{1}, X_{2}, \ldots, X_{N(n)+M(n)}\right\} .
$$

Then, $\mathcal{P}_{n}^{-}$and $\mathcal{P}_{n}^{+}$are Poisson point processes on $\mathbb{R}^{d}$ with intensity functions $\left(n-n^{3 / 4}\right) f(\cdot)$ and $\left(n+n^{3 / 4}\right) f(\cdot)$, respectively. The point processes $\mathcal{P}_{n}^{-}$and $\mathcal{P}_{n}^{+}$are coupled in such a way that $\mathcal{P}_{n}^{-} \subseteq \mathcal{P}_{n}^{+}$. Furthermore, if $H_{n}=\left\{\mathcal{P}_{n}^{-} \subseteq \mathcal{X}_{n} \subseteq \mathcal{P}_{n}^{+}\right\}$then, by the Borel-Cantelli lemma and Lemma 3.2, $\mathrm{P}\left[H_{n}^{\mathrm{c}}\right.$ infinitely often $]=0$. Hence, a.s., the event $H_{n}$ happens eventually.

Fix $t \in(0,(d-1) / \alpha \lambda)$. Choose $u$ and $c$ such that $c<0, t<u<(d-1) / \alpha \lambda$, and $u<(c+\alpha(d-1)) / \alpha^{2} \lambda$. Pick $\varepsilon>0$ small enough such that $(1+\varepsilon) u<(c+\alpha(d-1)) / \alpha^{2} \lambda$ and $\varepsilon+t<u$. Fix an integer $a>1$, and let $v(m)=a^{m}, m=1,2, \ldots$ Define the annulus

$$
A_{m}(c)=B\left(0, R_{\nu(m)}(0)\right) \backslash B\left(0, R_{v(m)}(c)\right),
$$

where $R_{n}(c)$ is as defined in (3.1) (note that $R_{n}(c)<R_{n}(0)$ since $c<0$ ). Define the sequence of functions $\left\{\hat{r}_{m}(v)\right\}_{m \geq 1}$ by

$$
\hat{r}_{m}(v)= \begin{cases}r_{v(m)}(v) & \text { if } \alpha>1, \\ r_{v(m+1)}(v) & \text { if } \alpha \leq 1 .\end{cases}
$$

For each $m$, choose a nonrandom set $\left\{x_{1}^{m}, x_{2}^{m}, \ldots, x_{\sigma_{m}}^{m}\right\} \subset A_{m}(c)$, such that the balls

$$
B\left(x_{i}^{n}, \hat{r}_{m}(u)\right), \quad 1 \leq i \leq \sigma_{m},
$$

are disjoint. The packing number $\sigma_{m}$ is the maximum number of disjoint balls $B\left(x, \hat{r}_{m}(u)\right)$ with $x \in A_{m}(c)$. Using (3.4), we can find constants $c_{0}$ and $c_{1}$ such that, for all large $m$, we 
have

$$
\begin{aligned}
\sigma_{m} \geq c_{0} \frac{R_{v(m)}^{d}(0)-R_{v(m)}{ }^{d}(c)}{\hat{r}_{m}^{d}(u)} & \\
\geq & c_{1} \frac{R_{v(m)}^{d}(0)-R_{v(m)}^{d}(c)}{r_{v(m)}^{d}(u)} \\
=\frac{c_{1}}{(\lambda u)^{d}}\left(\frac{\log v(m)}{\log _{2} v(m)}\right)^{d}( & \left(1+\frac{d-\alpha}{\alpha} \frac{\log _{2} v(m)}{\log v(m)}\right)^{d / \alpha} \\
& \left.\quad-\left(1+\frac{c+d-\alpha}{\alpha} \frac{\log _{2} v(m)}{\log v(m)}\right)^{d / \alpha}\right) .
\end{aligned}
$$

The function $g(x)=(1+x(d-\alpha) / \alpha)^{d / \alpha}-(1+x(c+d-\alpha) / \alpha)^{d / \alpha}, x \geq 0$, can satisfy $g(0)=0$ and $g^{\prime}(0)=-c d / \alpha^{2}>0$, since $c<0$. Hence, for all sufficiently small $x>0$, we have $g(x) \geq \delta x$ for some constant $\delta>0$. Using this inequality in the above lower bound for $\sigma_{m}$, we obtain

$$
\sigma_{m} \geq c_{2}\left(\frac{\log v(m)}{\log _{2} v(m)}\right)^{d-1}
$$

for large $m$ and some constant $c_{2}$. By part 2 of Lemma 3.1, there will a.s. be points of $\mathcal{P}_{\nu(m)}^{-}$in $A_{m}$ for all large enough $m$. Consider the sequence of sets $\left(\bigcup_{i=1}^{\sigma_{m}} E_{m, i}\right)^{\mathrm{c}}$, where

$$
E_{m, i}=\left\{\mathcal{P}_{\nu(m)}^{-}\left[B\left(x_{i}^{\nu(m)}, \hat{r}_{m}(\varepsilon)\right)\right]=1\right\} \cap\left\{\mathcal{P}_{\nu(m+1)}^{+}\left[B\left(x_{i}^{\nu(m)}, \hat{r}_{m}(u)\right)\right]=1\right\}
$$

for $i=1,2, \ldots, \sigma_{m}, m=1,2, \ldots$ From an earlier argument $\mathrm{P}\left[H_{n}^{\mathrm{c}}\right]$ is summable and, hence, with probability $1, H_{n}$ happens eventually. For any $n>a$, let $m$ be such that $a^{m} \leq$ $n \leq a^{m+1}$. Recall that $\{N(n)\}_{n \geq 1}$ and $\{M(n)\}_{n \geq 1}$ are nondecreasing. Hence, if $H_{n}$ and $E_{m, i}$ happen, then there is a point $X \in \mathcal{P}_{\nu(m)}^{-} \subset \mathcal{P}_{n}^{-} \subset \mathcal{X}_{n}$ such that $X \in B\left(x_{i}^{\nu(m)}, \hat{r}_{m}(\varepsilon)\right)$ with no other point of $\mathcal{P}_{v(m+1)}^{+} \supset \mathcal{P}_{n}^{+}$(and, hence, of $\mathcal{X}_{n}$ ) in $B\left(x_{i}^{\nu(m)}, \hat{r}_{m}(u)\right.$ ). This would imply that $d_{n} \geq \hat{r}_{m}(u)-\hat{r}_{m}(\varepsilon) \geq \hat{r}_{m}(t) \geq r_{n}(t)$. Thus, by the Borel-Cantelli lemma, the proof of Proposition 3.2 is complete if we show that

$$
\sum_{m=1}^{\infty} \mathrm{P}\left[\left(\bigcup_{i=1}^{\sigma_{m}} E_{m, i}\right)^{\mathrm{c}}\right]<\infty .
$$

To this end, we first estimate $\mathrm{P}\left[E_{m, i}\right]$. For $i=1,2, \ldots, \sigma_{m}, m=1,2, \ldots$, define the sets

$$
\ell_{m}=\mathcal{P}_{\nu(m+1)}^{+} \backslash \mathcal{P}_{\nu(m)}^{-}, \quad U_{m, i}=B\left(x_{i}^{\nu(m)}, \hat{r}_{m}(\varepsilon)\right), \quad V_{m, i}=B\left(x_{i}^{\nu(m)}, \hat{r}_{m}(u)\right) \backslash U_{m, i}
$$

Then, $E_{m, i}=\left\{\mathcal{P}_{\nu(m)}^{-}\left[U_{m, i}\right]=1\right\} \cap\left\{\mathcal{P}_{\nu(m)}^{-}\left[V_{m, i}\right]=0\right\} \cap\left\{\ell_{m}\left[U_{m, i}\right]=0\right\} \cap\left\{\ell_{m}\left[V_{m, i}\right]=0\right\}$. Let $\alpha(m)=v(m)-v(m)^{3 / 4}$ and $\beta(m)=v(m+1)+v(m+1)^{3 / 4}$. Note that each of the four events appearing in the above equation are independent. Hence,

$$
\begin{aligned}
\mathrm{P}\left[E_{m, i}\right] & =\left(\alpha(m) \int_{U_{m, i}} f(y) \mathrm{d} y\right) \exp \left(-\beta(m) \int_{U_{m, i} \cup V_{m, i}} f(y) \mathrm{d} y\right) \\
& =\alpha(m) I\left(x_{i}^{\nu(m)}, \hat{r}_{m}(\varepsilon)\right) \exp \left(-\beta(m) I\left(x_{i}^{v(m)}, \hat{r}_{m}(u)\right)\right) \\
& \geq \alpha(m) I\left(R_{v(m)}(0), \hat{r}_{m}(\varepsilon)\right) \exp \left(-\beta(m) I\left(R_{v(m)}(c), \hat{r}_{m}(u)\right)\right),
\end{aligned}
$$


where the last inequality follows since $R_{\nu(m)}(c) \leq\left|x_{i}^{\nu(m)}\right| \leq R_{\nu(m)}(0)$ and the density is decreasing radially. Using Lemma 2.1 (that this lemma is applicable is shown in the proof of Proposition 3.1; see the arguments below (3.11) leading to (3.12)) and noting that $\alpha(m) \sim v(m)$ and $\beta(m) \sim a v(m)$, we can find constants $C_{1}^{\prime}$ and $C_{2}^{\prime}$, such that, for large $m$,

$$
\begin{aligned}
\mathrm{P}\left[E_{m, i}\right] \geq & C_{1}^{\prime} v(m) \hat{r}_{m}^{(d-1) / 2}(\varepsilon) \exp \left(-\lambda\left(R_{v(m)}^{\alpha}(0)-\alpha \hat{r}_{m}(\varepsilon) R_{v(m)}^{\alpha-1}(0)\right)\right)\left(R_{v(m)}^{\alpha-1}(0)\right)^{-(d+1) / 2} \\
& \times \exp \left(-C_{2}^{\prime} v(m) \hat{r}_{m}^{(d-1) / 2}(u) \exp \left(-\lambda\left(R_{v(m)}^{\alpha}(c)-\alpha \hat{r}_{m}(u) R_{v(m)}^{\alpha-1}(c)\right)\right)\right. \\
& \left.\times\left(R_{v(m)}^{\alpha-1}(c)\right)^{-(d+1) / 2}\right)
\end{aligned}
$$

We now estimate the right-hand side of the above inequality. In what follows, we will use (3.4) to obtain the desired inequalities for the $\alpha \leq 1$ case, as in the proof of Proposition 3.1, without mentioning it explicitly. From (3.15), with $c=0$ and $u$ replaced by $\varepsilon$, we obtain $\lambda \alpha \hat{r}_{m}(\varepsilon) R_{\nu(m)}^{\alpha-1}(0) \geq \log _{2}(v(m)) \lambda \alpha \varepsilon / 2$ for large $m$. Hence, we can find a constant $c_{3}^{\prime}$ such that, for large $m$,

$$
\exp \left(\lambda \alpha \hat{r}_{m}(\varepsilon) R_{\nu(m)}^{\alpha-1}(0)\right)\left(\hat{r}_{m}(\varepsilon)\right)^{(d-1) / 2} \geq c_{3}^{\prime} \frac{\mathrm{e}^{\log _{2} \nu(m) \lambda \alpha \varepsilon / 2}\left(\log _{2} v(m)\right)^{(d-1) / 2}}{(\log v(m))^{(1-1 / \alpha)(d-1) / 2}} .
$$

Using (3.14) with $c=0$ and the above inequality, we obtain, for large $m$ and some constant $C_{3}^{\prime}$,

$$
\begin{aligned}
& C_{1}^{\prime} v(m) \hat{r}_{m}^{(d-1) / 2}(\varepsilon) \exp \left(-\lambda\left(R_{v(m)}^{\alpha}(0)-\alpha \hat{r}_{m}(\varepsilon) R_{\nu(m)}^{(\alpha-1)}(0)\right)\right)\left(R_{\nu(m)}^{(\alpha-1)}(0)\right)^{-(d+1) / 2} \\
& \quad \geq C_{3}^{\prime} v(m) \frac{(\log v(m))^{-(1-1 / \alpha)(d+1) / 2}}{v(m)(\log v(m))^{(d-\alpha) / \alpha}} \frac{\mathrm{e}^{\log _{2} v(m) \lambda \alpha \varepsilon / 2}\left(\log _{2} v(m)\right)^{(d-1) / 2}}{(\log v(m))^{(1-1 / \alpha)(d-1) / 2}} \\
& \quad=C_{3}^{\prime} \frac{\left(\log _{2} v(m)\right)^{(d-1) / 2}}{(\log v(m))^{d-1-\alpha \varepsilon \lambda / 2}} .
\end{aligned}
$$

From (3.15), we obtain, for large $m, \lambda \alpha \hat{r}_{m}(u) R_{v(m)}^{\alpha-1}(c) \leq(1+\varepsilon) \lambda \alpha u \log _{2} v(m)$, from which we obtain, for some constant $C_{4}^{\prime}$,

$$
\begin{aligned}
\exp \left(\lambda \alpha \hat{r}_{m}(u) R_{v(m)}^{\alpha-1}(c)\right)\left(\hat{r}_{m}(u)\right)^{(d-1) / 2} & \leq C_{4}^{\prime} \frac{\mathrm{e}^{(1+\varepsilon) \lambda \alpha u \log _{2} v(m)}\left(\log _{2} v(m)\right)^{(d-1) / 2}}{(\log v(m))^{(1-1 / \alpha)(d-1) / 2}} \\
& =C_{4}^{\prime} \frac{(\log v(m))^{(1+\varepsilon) \lambda \alpha u}\left(\log _{2} v(m)\right)^{(d-1) / 2}}{(\log v(m))^{(1-1 / \alpha)(d-1) / 2}}
\end{aligned}
$$

As in (3.14), we can find a constant $C_{5}^{\prime}$ such that, for large $m$,

$$
\exp \left(-\lambda R_{v(m)}^{\alpha}(c)\right)\left(R_{v(m)}^{\alpha-1}(c)\right)^{-(d+1) / 2} \leq C_{5}^{\prime} \frac{(\log v(m))^{-(1-1 / \alpha)(d+1) / 2}}{v(m)(\log v(m))^{(c+d-\alpha) / \alpha}} .
$$

Using the two bounds obtained above, we obtain, for large $m$ and some constant $C_{6}^{\prime}$,

$$
\begin{aligned}
& C_{2}^{\prime} v(m) \hat{r}_{m}^{(d-1) / 2}(u) \exp \left(-\lambda\left(R_{v(m)}^{\alpha}(c)-\alpha \hat{r}_{m}(u) R_{v(m)}^{\alpha-1}(c)\right)\right)\left(R_{v(m)}^{\alpha-1}(c)\right)^{-(d+1) / 2} \\
& \quad \leq C_{6}^{\prime} \frac{\left(\log _{2}(v(m))\right)^{(d-1) / 2}}{(\log (v(m)))^{d+c / \alpha-(1+\varepsilon) \alpha u \lambda-1}} .
\end{aligned}
$$


Using (3.27) and the above inequality in (3.26), we obtain, for large $m$,

$$
\begin{aligned}
\mathrm{P}\left[E_{m, i}\right] & \geq C_{3}^{\prime} \frac{\left(\log _{2}(v(m))\right)^{(d-1) / 2}}{(\log (v(m)))^{d-1-\alpha \varepsilon \lambda / 2}} \exp \left(-C_{6}^{\prime} \frac{\left(\log _{2}(v(m))\right)^{(d-1) / 2}}{(\log (v(m)))^{d+c / \alpha-(1+\varepsilon) \lambda \alpha u-1}}\right) \\
& \sim C_{3}^{\prime} \frac{\left(\log _{2}(v(m))\right)^{(d-1) / 2}}{(\log (v(m)))^{d-1-\alpha \varepsilon \lambda / 2}},
\end{aligned}
$$

where the last relation follows since $(1+\varepsilon) u<(\alpha d+c-\alpha) / \alpha^{2} \lambda$. The events $E_{m, i}, 1 \leq i \leq \sigma_{m}$, are independent, since the balls $B\left(x_{i}^{\nu(m)}, \hat{r}_{m}(u)\right)$ are disjoint. So, by (3.23), (3.28), and the inequality $1-x \leq \mathrm{e}^{-x}$, we can find constants $C^{\prime}$ and $C^{\prime \prime}$ such that, for all large enough $m$,

$$
\begin{aligned}
\mathrm{P}\left[\left(\bigcup_{i=1}^{\sigma_{m}} E_{m, i}\right)^{\mathrm{c}}\right] & \leq \prod_{i=1}^{\sigma_{m}} \exp \left(-\mathrm{P}\left[E_{m, i}\right]\right) \\
& \leq \exp \left(-C^{\prime} \sigma_{m} \frac{\left(\log _{2}(\nu(m))\right)^{(d-1) / 2}}{(\log (\nu(m)))^{d-1-\alpha \varepsilon \lambda / 2}}\right) \\
& \leq \exp \left(-C^{\prime \prime}\left(\frac{m}{\log m+\log _{2} a}\right)^{d-1} \frac{\left(\log m+\log _{2} a\right)^{(d-1) / 2}}{m^{d-1-\alpha \varepsilon \lambda / 2}}\right) \\
& =\exp \left(-C^{\prime \prime} \frac{m^{\alpha \varepsilon \lambda / 2}}{\left(\log m+\log _{2} a\right)^{(d-1) / 2}}\right),
\end{aligned}
$$

which is summable in $m$. This proves (3.24).

Proof of Theorem 1.3. The proof is immediate from Propositions 3.1 and 3.2.

\section{Appendix A. Proof of Lemma 2.1}

In the definition of $I\left(\rho_{n}, r_{n}\right)=I\left(\rho_{n} e, r_{n}\right)$, set $y=\left(\rho_{n}+r_{n} t, r_{n} s\right), t \in(-1,1), s \in \mathbb{R}^{d-1}$. This gives

$$
I\left(\rho_{n}, r_{n}\right)=A_{d} \int_{-1}^{1} \int_{\|s\|^{2} \leq\left(1-t^{2}\right), s \in \mathbb{R}^{d-1}} \exp \left(-\lambda\left(\left(\rho_{n}+r_{n} t\right)^{2}+\left(\|s\| r_{n}\right)^{2}\right)^{\alpha / 2}\right) r_{n}^{d} \mathrm{~d} s \mathrm{~d} t
$$

First consider the case in which $0<\alpha \leq 2$. Using the Taylor expansion, we obtain

$$
\begin{aligned}
\left(\left(\rho_{n}+r_{n} t\right)^{2}+\left(\|s\| r_{n}\right)^{2}\right)^{\alpha / 2} & =\left(\left(\rho_{n}^{2}+2 r_{n} t \rho_{n}\right)+\left(t^{2}+\|s\|^{2}\right) r_{n}^{2}\right)^{\alpha / 2} \\
& =\left(\rho_{n}^{2}+2 r_{n} \rho_{n} t\right)^{\alpha / 2}+h_{1}(n, s, t),
\end{aligned}
$$

where $h_{1}(n, s, t)=(\alpha / 2) r_{n}^{2}\left(t^{2}+\|s\|^{2}\right)\left(\rho_{n}^{2}+2 r_{n} \rho_{n} t+\xi\right)^{\alpha / 2-1}$ and $\xi \in\left(0, r_{n}^{2}\left(t^{2}+\|s\|^{2}\right)\right)$. Since $0<\alpha \leq 2,(t, s) \in B(0,1)$, and $0 \leq \xi \leq r_{n}^{2}$, we have

$$
0 \leq h_{1}(n, s, t) \leq \frac{\alpha}{2} r_{n}^{2}\left(t^{2}+\|s\|^{2}\right)\left(\rho_{n}^{2}+2 r_{n} \rho_{n} t\right)^{\alpha / 2-1} \leq w_{1}(n),
$$

where

$$
0 \leq w_{1}(n)=\frac{\alpha}{2} r_{n}^{2}\left(\rho_{n}^{2}-2 r_{n} \rho_{n}\right)^{\alpha / 2-1}=\frac{\alpha}{2} r_{n}^{2} \rho_{n}^{\alpha-2}\left(1-\frac{2 r_{n}}{\rho_{n}}\right)^{\alpha / 2-1} \rightarrow 0,
$$


since $r_{n}^{2} \rho_{n}^{\alpha-2} \rightarrow 0$, and $r_{n} / \rho_{n} \rightarrow 0$ as $n \rightarrow \infty$. Again, from the Taylor expansion applied to $\left(\rho_{n}^{2}+2 r_{n} \rho_{n} t\right)^{\alpha / 2}$ in (A.2), we obtain

$$
\left(\rho_{n}^{2}+2 r_{n} \rho_{n} t\right)^{\alpha / 2}=\rho_{n}^{\alpha}+\alpha r_{n} t \rho_{n}^{\alpha-1}+h_{2}(n, t),
$$

where $h_{2}(n, t)=\frac{1}{2} \alpha(\alpha-2)\left(r_{n} t \rho_{n}\right)^{2}\left(\rho_{n}^{2}+\zeta\right)^{\alpha / 2-2}$ and $\zeta \in\left(\min \left(0,2 \rho_{n} r_{n} t\right), \max \left(0,2 \rho_{n} r_{n} t\right)\right)$. Since $0<\alpha \leq 2$ and $-1 \leq t \leq 1$, we obtain

$$
w_{2}(n)=\frac{\alpha(\alpha-2)}{2} r_{n}^{2} \rho_{n}^{\alpha-2}\left(1-2 \frac{r_{n}}{\rho_{n}}\right)^{\alpha / 2-2} \leq h_{2}(n, t) \leq 0 .
$$

Since $r_{n}^{2} \rho_{n}^{\alpha-2} \rightarrow 0$ and $r_{n} / \rho_{n} \rightarrow 0$, it follows that $w_{2}(n) \rightarrow 0$ as $n \rightarrow \infty$. From (A.2)-(A.5), we obtain

$$
\rho_{n}^{\alpha}+\alpha r_{n} t \rho_{n}^{\alpha-1}+w_{2} \leq\left(\left(\rho_{n}+r_{n} t\right)^{2}+\left(\|s\| r_{n}\right)^{2}\right)^{\alpha / 2} \leq \rho_{n}^{\alpha}+\alpha r_{n} t \rho_{n}^{\alpha-1}+w_{1} .
$$

Using the above in (A.1), we obtain

$$
A_{d} r_{n}^{d} \mathrm{e}^{-\lambda w_{1}} G_{n} \leq I\left(\rho_{n}, r_{n}\right) \leq A_{d} r_{n}^{d} \mathrm{e}^{-\lambda w_{2}} G_{n}
$$

where

$$
G_{n}=\int_{-1}^{1} \int_{\|s\|^{2} \leq\left(1-t^{2}\right), s \in \mathbb{R}^{d-1}} \exp \left(-\lambda\left(\rho_{n}^{\alpha}+\alpha r_{n} t \rho_{n}^{\alpha-1}\right)\right) \mathrm{d} s \mathrm{~d} t,
$$

and $w_{1}$ and $w_{2}$, as defined in (A.3) and (A.5), respectively, converge to 0 as $n \rightarrow \infty$.

If $\alpha>2$ then $h_{2}(n, t) \geq 0$, and we take $w_{1}$ and $w_{2}$ to be the upper and lower bounds of $h_{1}(n, s, t)+h_{2}(n, t)$, respectively. We then obtain (A.6) with $w_{2}(n)=0$, and

$$
\begin{aligned}
w_{1}(n) & =\frac{\alpha}{2} r_{n}^{2}\left(\rho_{n}^{2}+2 r_{n} \rho_{n}\right)^{\alpha / 2-1}\left[1+(\alpha-2) \rho_{n}^{2}\left(\rho_{n}^{2}-2 r_{n} \rho_{n}\right)^{-1}\right] \\
& =\frac{\alpha}{2} r_{n}^{2} \rho_{n}^{\alpha-2}\left(1+2 \frac{r_{n}}{\rho_{n}}\right)^{\alpha / 2-1}\left[1+(\alpha-2)\left(1-2 \frac{r_{n}}{\rho_{n}}\right)^{-1}\right],
\end{aligned}
$$

which converges to 0 by the conditions of the lemma. Now consider the integral in (A.7). First make the change of variable $u=t+1$ and then set $v=\lambda \alpha r_{n} \rho_{n}^{\alpha-1} u$ to obtain

$$
\begin{aligned}
G_{n} & =\theta_{d-1} \mathrm{e}^{-\lambda \rho_{n}^{\alpha}} \int_{-1}^{1} \exp \left(-\lambda \alpha r_{n} \rho_{n}^{\alpha-1} t\right)\left(1-t^{2}\right)^{(d-1) / 2} \mathrm{~d} t \\
& =\theta_{d-1} \exp \left(-\lambda\left(\rho_{n}^{\alpha}-\alpha r_{n} \rho_{n}^{\alpha-1}\right)\right) \int_{0}^{2} \exp \left(-\lambda \alpha r_{n} \rho_{n}^{\alpha-1} u\right) u^{(d-1) / 2}(2-u)^{(d-1) / 2} \mathrm{~d} u \\
& =\theta_{d-1} \exp \left(-\lambda\left(\rho_{n}^{\alpha}-\alpha r_{n} \rho_{n}^{\alpha-1}\right)\right)\left(\lambda \alpha r_{n} \rho_{n}^{\alpha-1}\right)^{-(d+1) / 2} 2^{(d-1) / 2} M_{n},
\end{aligned}
$$

where

$$
M_{n}=\int_{0}^{2 \lambda \alpha r_{n} \rho_{n}^{\alpha-1}} \mathrm{e}^{-v} v^{(d-1) / 2}\left(1-\frac{v}{2 \lambda \alpha r_{n} \rho_{n}^{\alpha-1}}\right)^{(d-1) / 2} \mathrm{~d} v \leq \Gamma\left(\frac{d+1}{2}\right) .
$$

We will show that the integral in (A.9) converges to $\Gamma((d+1) / 2)$ as $n \rightarrow \infty$, and also 
estimate the error in this approximation. Note that $r_{n} \rho_{n}^{\alpha-1} \rightarrow \infty$ as $n \rightarrow \infty$. Write $E_{n}=$ $M_{n}-\Gamma((d+1) / 2)=A_{n}-B_{n}$, where

$$
\begin{aligned}
& A_{n}=\int_{0}^{2 \lambda \alpha r_{n} \rho_{n}^{\alpha-1}} \mathrm{e}^{-v} v^{(d-1) / 2}\left[\left(1-\frac{v}{2 \lambda \alpha r_{n} \rho_{n}^{\alpha-1}}\right)^{(d-1) / 2}-1\right] \mathrm{d} v, \\
& B_{n}=\int_{2 \lambda \alpha r_{n} \rho_{n}^{\alpha-1}}^{\infty} \mathrm{e}^{-v} v^{(d-1) / 2} \mathrm{~d} v,
\end{aligned}
$$

and

$$
\left|A_{n}\right| \leq \sup _{0 \leq v \leq 2 \lambda \alpha r_{n} \rho_{n}^{\alpha-1}}\left\{\mathrm{e}^{-v / 2}\left|1-\left(1-\frac{v}{2 \lambda \alpha r_{n} \rho_{n}^{\alpha-1}}\right)^{(d-1) / 2}\right|\right\} \int_{0}^{\infty} \mathrm{e}^{-v / 2} v^{(d-1) / 2} \mathrm{~d} v .
$$

Since $(1-x)^{a} \geq 1-C x, 0 \leq x \leq 1$, with $C=\mathbf{1}_{\{0<a \leq 1\}}+a \mathbf{1}_{\{a>1\}}$, we obtain

$$
0 \leq 1-\left(1-\frac{v}{2 \lambda \alpha r_{n} \rho_{n}^{\alpha-1}}\right)^{(d-1) / 2} \leq \frac{C v}{2 \lambda \alpha r_{n} \rho_{n}^{\alpha-1}}, \quad 0 \leq v \leq 2 \lambda \alpha r_{n} \rho_{n}^{\alpha-1} .
$$

Therefore,

$$
\left|A_{n}\right| \leq \frac{C}{2 \lambda \alpha r_{n} \rho_{n}^{\alpha-1}} \sup _{0 \leq v<\infty}\left\{v \mathrm{e}^{-v / 2}\right\} \int_{0}^{\infty} \mathrm{e}^{-v / 2} v^{(d-1) / 2} \mathrm{~d} v=\frac{C^{\prime}}{r_{n} \rho_{n}^{\alpha-1}},
$$

where $C^{\prime}$ is some constant. Furthermore, $\left|B_{n}\right| \leq \exp \left(-\lambda \alpha r_{n} \rho_{n}^{\alpha-1} / 2\right) \int_{0}^{\infty} \mathrm{e}^{-v / 2} v^{(d-1) / 2} \mathrm{~d} v$, and, hence, decays exponentially fast in $r_{n} \rho_{n}^{\alpha-1}$. Putting the above two estimates together, we obtain

$$
\left|E_{n}\right| \leq \frac{C_{1}}{r_{n} \rho_{n}^{\alpha-1}} \rightarrow 0 \quad \text { as } n \rightarrow \infty .
$$

The result now follows from (A.6), (A.8), and (A.10).

\section{Acknowledgement}

The authors would like to thank an anonymous referee for numerous suggestions that significantly improved this paper.

\section{References}

Appel, M. J. B. AND Russo, R. P. (1997). The minimum vertex degree of a graph on the uniform points in $[0,1]^{d}$. Adv. Appl. Prob. 29, 582-594.

Dette, H. and Henze, N. (1989). The limit distribution of the largest nearest-neighbour link in the unit $d$-cube. J. Appl. Prob. 26, 67-80.

Gupta, B., Iyer, S. K. And Manjunath, D. (2005). On the topological properties of one dimensional exponential random geometric graphs. Random Structures and Algorithms 32, 181-204.

Hsing, T. and Rootzén, H. (2005). Extremes on trees. Ann. Prob. 33, 413-444.

Penrose, M. D. (1997). The longest edge of the minimal spanning tree. Ann. Appl. Prob. 7, 340-361.

Penrose, M. D. (1998). Extremes for the minimal spanning tree on normally distributed points. Adv. Appl. Prob. 30, 628-639.

Penrose, M. D. (1999). A strong law for the largest nearest-neighbour link between random points. J. London Math. Soc. 60, 951-960.

Penrose, M. (2003). Random Geometric Graphs. Oxford University Press.

Steele, J. M. And Tierney, L. (1986). Boundary domination and the distribution of the largest nearest-neighbor link in higher dimensions. J. Appl. Prob. 23, 524-528. 